\title{
Implications of light $Z^{\prime}$ on semileptonic $B\left(B_{s}\right) \rightarrow T\left\{K_{2}^{*}(1430)\left(f_{2}^{\prime}(1525)\right)\right\} \ell^{+} \ell^{-}$decays at large recoil
}

\author{
Manas K. Mohapatra $\odot^{*}$ and Anjan Giri $\odot^{\dagger}$ \\ Department of Physics, IIT Hyderabad, Kandi 502285, India
}

(Received 3 October 2021; accepted 18 October 2021; published 12 November 2021)

\begin{abstract}
We probe the rare semileptonic decays $B_{(s)} \rightarrow K_{2}^{*}(1430)\left(f_{2}^{\prime}(1525)\right) \ell^{+} \ell^{-}$proceeding via $b \rightarrow s \ell \ell$ transition in the presence of a light $Z^{\prime}$ boson. We employ the presence of an additional vector-type interaction and constrain the new physics coupling parameter using the existing experimental measurements on $R_{K}$ and $R_{K^{*}}$ observables. To understand the sensitivity of the new physics coupling, we investigate the impact of this coupling on various physical observables such as differential branching ratio, the forward-backward asymmetry, the lepton polarization asymmetry, the angular observable $P_{5}^{\prime}$, and the lepton universality parameters such as the ratio of the branching ratio $R_{f_{2}^{\prime}\left(K_{2}^{*}\right)}$ and some important Q parameters of $B_{(s)} \rightarrow K_{2}^{*}(1430)\left(f_{2}^{\prime}(1525)\right) \ell^{+} \ell^{-}$processes at large recoil. We find some noticeable differences of the observables in the presence of light $Z^{\prime}$ contribution.
\end{abstract}

DOI: 10.1103/PhysRevD.104.095012

\section{INTRODUCTION}

According to our best understanding, the standard model (SM), although a successful theory, is not enough to explain some key puzzles such as matter-antimatter asymmetry in the Universe, dark matter, dark energy, hierarchy problem, neutrino mass, and so on. Hunting for beyond the SM has been a challenge to the whole high-energy physics community. To understand the nature, the flavor physics, in principle, could be the ideal platform to explore the new physics (NP) beyond the SM. In this respect, the ongoing endeavor in B meson decays is of great interest in testing the SM and shedding light on the NP beyond it. However, in recent years, a few measurements in rare weak decays of the B meson have shown deviations from the SM predictions both in flavor changing neutral current (FCNC) which undergo $b \rightarrow$ sl $\ell$ parton level and in flavor changing charged current mediated by $b \rightarrow c \ell \nu$ transition. In the light of neutral quark-level transitions, several measurements, most importantly, the lepton flavor universality violation (LFUV) parameter $R_{K^{*}}=\mathcal{B R}\left(B \rightarrow K^{*} \mu^{+} \mu^{-}\right) /$ $\mathcal{B R}\left(B \rightarrow K^{*} e^{+} e^{-}\right)$observed from LHCb [1,2] and Belle [3], have $2.1-2.4 \sigma$ deviation from SM prediction $\sim 1[4,5]$. However, recently the measurement of another clean observable $R_{K}=\mathcal{B R}\left(B \rightarrow K \mu^{+} \mu^{-}\right) / \mathcal{B R}\left(B \rightarrow K e^{+} e^{-}\right)[4-6]$ has

\footnotetext{
*manasmohapatra12@gmail.com

†iria@iith.ac.in
}

Published by the American Physical Society under the terms of the Creative Commons Attribution 4.0 International license. Further distribution of this work must maintain attribution to the author(s) and the published article's title, journal citation, and DOI. Funded by SCOAP ${ }^{3}$. been observed in the dilepton invariant mass-squared range $1.1 \leq q^{2} \leq 6.0 \mathrm{GeV}^{2}$ from the $\mathrm{LHCb}$ experiment which indicates $3.1 \sigma$ discrepancy [7]. The experimental measurements of $R_{K}$ and $R_{K^{*}}$ are given as follows:

$$
\begin{array}{ll}
R_{K}^{\operatorname{Exp}}=0.846_{-0.846-0.042}^{+0.013+0.039}, & 1.1 \leq q^{2} \leq 6.0 \mathrm{GeV}^{2}, \\
R_{K^{*}}^{\operatorname{Exp}}=0.660_{-0.07}^{+0.11} \pm 0.03, & 0.045 \leq q^{2} \leq 1.1 \mathrm{GeV}^{2} \quad\left(\operatorname{low} q^{2}\right), \\
R_{K^{*}}^{\operatorname{Exp}}=0.690_{-0.07}^{+0.11} \pm 0.05, & 1.1 \leq q^{2} \leq 6.0 \mathrm{GeV}^{2}\left(\operatorname{central} q^{2}\right) .
\end{array}
$$

Similarly, another anomaly, the so-called angular observable $P_{5}^{\prime}$ in $B \rightarrow K^{*} \mu^{+} \mu^{-}$decay mode observed from LHCb [8,9], ATLAS [10], CMS [11], and Belle [12] Collaborations, contributes (1-4) $\sigma$ deviations from the SM expectation $[13,14]$. Furthermore, a $3.6 \sigma$ deviation is seen in the branching ratio of $B_{s} \rightarrow \phi \ell \ell$ process in the $q^{2} \in[1.1,6.0]$ region by $\mathrm{LHCb}[15,16]$.

Decays of B mesons to S-wave mesons (pseudoscalar and vector mesons) have been explored widely in both theory as well as experiment, whereas the analysis of the P-wave mesons (scalar, axial vector, and tensor mesons) in B decays has got relatively less attention. However, it is observed that a large number of such decays have been established experimentally [17]. Therefore, in this work we intend to investigate the semileptonic decays of $\mathrm{B}$ mesons into light $\mathrm{P}$ wave tensor (T) mesons with $J^{P}=2^{+}$containing $f_{2}^{\prime}(1525)$ and $K_{2}^{*}(1430)$ in the final state. The decay mode $B \rightarrow$ $K_{2}^{*} \ell^{+} \ell^{-}$has been discussed in Refs. [18-25]. Similarly, in Ref. [26], though the authors have investigated the NP effect in the presence of both a vectorlike quark model and a family 
nonuniversal $Z^{\prime}$ model, unfortunately less emphasis was offered to the $B_{s} \rightarrow f_{2}^{\prime} \ell^{+} \ell^{-}$process. However, recently a detailed angular analysis of $B_{s} \rightarrow f_{2}^{\prime} \ell^{+} \ell^{-}$decay has been studied in the context of effective field theory framework [27]. In this work, we are not considering the branching ratios of $f_{2}^{\prime}$ and $K_{2}^{*}$ tensors in the given $B_{s} \rightarrow f_{2}^{\prime} \ell^{+} \ell^{-}$and $B \rightarrow K_{2}^{*} \ell^{+} \ell^{-}$processes, respectively. In the theoretical calculations, the knowledge of nonperturbative QCD is necessary which is parameterized in terms of decay constant, form factors. The form factors for $B_{(s)} \rightarrow T$ transition have been calculated in the Isgur-Scora-Grinstein-Wise quark model (ISGW) [28] and in the ISGW2 model [29,30], the perturbative QCD method [31], and the light-cone sum rule (LCSR) approach [32].

Since the branching ratio includes the hadronic uncertainties unlike the clean observables $R_{K}$ and $R_{K^{*}}$, the NP is allowed in the muon and/or electron mode in $b \rightarrow s \ell^{+} \ell^{-}$ quark-level transition. Mostly, in several works the authors have analyzed with a heavy mediator such as heavy $Z^{\prime}$ leptoquarks [33-42] in the physics beyond the SM. However, in the presence of light mediators, the discrepancy can also be explained for the observables like the $R_{K}$ and $R_{K^{*}}$ [43-45]. In this respect, we consider a light $Z^{\prime}$ in which the NP Wilson coefficients are $q^{2}$ dependent $[33,38,44-46]$ and study the impact on $B\left(B_{s}\right) \rightarrow T\left\{K_{2}^{*}(1430)\left(f_{2}^{\prime}(1525)\right)\right\} \ell^{+} \ell^{-}$decays.

The organization of the paper is as follows. In Sec. II, we deliver the theoretical formalism that includes a brief review of generalized weak effective Hamiltonian for $b \rightarrow s \ell^{+} \ell^{-}$FCNC transition. Additionally, we also present the $B \rightarrow T$ hadronic matrix elements. We provide the formulas of differential branching ratios and other observables of $B_{s} \rightarrow f_{2}^{\prime} \ell^{+} \ell^{-}$and $B \rightarrow K_{2}^{*} \ell^{+} \ell^{-}$processes in Sec. III. In Sec. IV, we analyze the NP contribution in the presence of the light $Z^{\prime}$ model. In Sec. V, we discuss and analyze our results in the presence of new physics. To conclude, we provide a brief summary of our results in Sec. VI.

\section{FORMALISM}

\section{A. Generalized effective weak Hamiltonian}

The generalized effective weak Hamiltonian for rare $b \rightarrow s \ell^{+} \ell^{-}(|\Delta B|=|\Delta S|=1)$ transition is given as $[47,48]$

$$
\begin{aligned}
\mathcal{H}_{\mathrm{eff}}= & -\frac{G_{F}}{\sqrt{2}} V_{t b} V_{t s}^{*} \frac{\alpha}{4 \pi}\left[C_{9}^{\mathrm{eff}} \bar{s} \gamma^{\mu} P_{L} b \bar{l} \gamma_{\mu} l+C_{10}^{\mathrm{eff}} \bar{s} \gamma^{\mu} P_{L} b \bar{l} \gamma_{\mu} \gamma_{5} l\right. \\
& \left.-\frac{2 m_{b}}{q^{2}} C_{7}^{\mathrm{eff}} \bar{s} i q_{\nu} \sigma^{\mu \nu} P_{R} b \bar{l} \gamma_{\mu} l\right]
\end{aligned}
$$

where $G_{F}$ is the Fermi coupling constant, $V_{i j}$ are the Cabibbo-Kobayashi-Maskawa (CKM) matrix element, $\alpha$ is the fine structure constant, $P_{L(R)}$ is the left (right) chiral project operator, and $F_{\mu \nu}$ is the electromagnetic field strength tensor. The factorizable loop terms can be explained in terms of $C_{7}^{\text {eff }}$ and $C_{9}^{\text {eff }}$ as [47]

$$
\begin{aligned}
C_{7}^{\mathrm{eff}}= & C_{7}-\frac{C_{5}}{3}-C_{6}, \\
C_{9}^{\mathrm{eff}}= & C_{9}(\mu)+h\left(\hat{m}_{c}, \hat{s}\right) C_{0}-\frac{1}{2} h(1, \hat{s}) \\
& \times\left(4 C_{3}+4 C_{4}+3 C_{5}+C_{6}\right)-\frac{1}{2} h(0, \hat{s})\left(C_{3}+3 C_{4}\right) \\
& +\frac{2}{9}\left(3 C_{3}+C_{4}+3 C_{5}+C_{6}\right),
\end{aligned}
$$

where $\hat{m}_{c}=m_{c} / m_{b}, \hat{s}=q^{2} / m_{b}^{2}$, and $C_{0}=3 C_{1}+C_{2}+$ $3 C_{3}+C_{4}+3 C_{5}+C_{6}$. The auxiliary functions given in the above equation are defined as

$$
\begin{aligned}
h(z, \hat{s})= & -\frac{8}{9} \ln \frac{m_{b}}{\mu}-\frac{8}{9} \ln z+\frac{8}{27}+\frac{4}{9} x-\frac{2}{9}(2+x)|1-x|^{1 / 2} \\
& \times \begin{cases}\ln \left|\frac{\sqrt{1-x}+1}{\sqrt{1-x}-1}\right|-i \pi, & \text { for } x \equiv \frac{4 z^{2}}{\hat{s}}<1, \\
2 \arctan \frac{1}{\sqrt{x-1}}, & \text { for } x \equiv \frac{4 z^{2}}{\hat{s}}>1,\end{cases} \\
& h(0, \hat{s})=-\frac{8}{9} \ln \frac{m_{b}}{\mu}-\frac{4}{9} \ln \hat{s}+\frac{8}{27}+\frac{4}{9} i \pi
\end{aligned}
$$

The effective Wilson coefficient $C_{9}^{\text {eff }}$ includes shortdistance contributions remain away from $c \bar{c}$ resonance zone, whereas the long-distance contributions which embed the resonant states $[J / \psi, \psi(2 S), \ldots]$ from $b \rightarrow c \bar{c} s\left(\rightarrow s \ell^{+} \ell^{-}\right)$are excluded in our present analysis. Therefore, we mainly dedicate to the $q^{2}$ rooms [0.045, $0.98]$ and $[1.1,6.0] \mathrm{GeV}^{2}$ only. However, we ignore the nonfactorizable corrections arising due to electromagnetic corrections to the hadronic matrix elements in the effective Hamiltonian in this work. Moreover, the $q^{2}$-dependent correction, i.e., the factorizable soft gluon part $\Delta C_{9}\left(q^{2}\right)$ coming from charm loop effects, is ignored in this work. However, the predicted ratio $\Delta C_{9}\left(q^{2}\right) / C_{9}$ has a significant contribution to $B \rightarrow K \ell \ell$ and $B \rightarrow K^{*} \ell \ell$, which is $\geq 5 \%$ and reaches up to $20 \%$, respectively [49]. In addition to this, recently in Ref. [50], the authors have presented the nonlocal contributions to $b \rightarrow s$ transition modes, i.e., $B \rightarrow K^{*}$ and $B_{s} \rightarrow \phi$ decays, where a modified analytic parameterization is proposed in the nonlocal matrix elements. However, this is very difficult to calculate, because it signs up the decay amplitude with nonperturbative nonlocal matrix elements. Therefore, we do not consider this effect in this work.

\section{B. $B \rightarrow T\left(K_{2}^{*}(1430), f_{2}^{\prime}(1525)\right)$ hadronic matrix elements}

A tensor $T$ meson of spin-2 state polarization can be established in terms of spin-1 polarization vectors [51]. The given tensor can be written symbolically as $\epsilon^{\mu \nu}(n)$, where 
" $n$ " corresponds to $0, \pm 1$, and \pm 2 . The explicit expressions are given as follows [26,31,51]:

$$
\begin{aligned}
\epsilon_{\mu \nu}(0) & =\frac{1}{\sqrt{6}}\left[\epsilon_{\mu}(+) \epsilon_{\nu}(-)+\epsilon_{\nu}(+) \epsilon_{\mu}(-)\right]+\sqrt{\frac{2}{3}} \epsilon_{\mu}(0) \epsilon_{\nu}(0), \\
\epsilon_{\mu \nu}( \pm 1) & =\frac{1}{\sqrt{2}}\left[\epsilon_{\mu}( \pm) \epsilon_{\nu}(0)+\epsilon_{\nu}( \pm) \epsilon_{\mu}(0)\right] \\
\epsilon_{\mu \nu}( \pm 2) & =\epsilon_{\mu}( \pm) \epsilon_{\nu}( \pm)
\end{aligned}
$$

where

$$
\epsilon_{\mu}(0)=\frac{1}{m_{T}}\left(E_{T}, 0,0, \vec{p}_{T}\right), \quad \epsilon_{\mu}( \pm)=\frac{1}{\sqrt{2}}(0, \mp 1,-i, 0) .
$$

Here, $m_{T}$ is the mass, and $E_{T}$ and $\vec{p}_{T}$ are the energy and momentum of the tensor meson in the $B$ meson rest frame, respectively. However, the information obtained from the helicity state for $n=2$ is not well understood of the finalstate two leptons. So the new polarization vector can be conveniently introduced as

$$
\epsilon_{T_{\mu}}(h)=\frac{1}{m_{B_{(s)}}} \epsilon_{\mu \nu}(h) P_{B_{(s)}}^{\nu},
$$

where $P_{B_{(s)}}^{\nu}$ is the four momentum of the $B_{(s)}$ meson. The expressions of the new polarization vectors $\epsilon_{T_{\mu}}(h)$ $(h=0, \pm 1, \pm 2)$ are given explicitly as [26]

$$
\begin{aligned}
\epsilon_{T_{\mu}}(0) & =\frac{1}{m_{B_{(s)}}} \sqrt{\frac{2}{3}} \epsilon(0) \cdot P_{B_{(s)}} \epsilon_{\mu}(0)=\frac{\sqrt{\lambda}}{\sqrt{6} m_{B_{s}} m_{T}} \epsilon_{\mu}(0), \\
\epsilon_{T_{\mu}}( \pm 1) & =\frac{1}{m_{B_{(s)}}} \frac{1}{\sqrt{2}} \epsilon(0) \cdot P_{B_{(s)}} \epsilon_{\mu}( \pm)=\frac{\sqrt{\lambda}}{\sqrt{8} m_{B_{(s)}} m_{T}} \epsilon_{\mu}( \pm), \\
\epsilon_{T_{\mu}}( \pm 2) & =0,
\end{aligned}
$$

where

$\lambda=m_{B_{(s)}}^{4}+m_{T}^{4}+q^{4}-2\left(m_{B_{(s)}}^{2} m_{T}^{2}+m_{B_{(s)}}^{2} q^{2}+q^{2} m_{T}^{2}\right)$.

The hadronic matrix elements of $B \rightarrow T$ transition, in analogy with $B \rightarrow V$, are given as $[31,32]$

$$
\begin{aligned}
\left\langle T\left(P_{T}, \epsilon\right)\left|(\bar{s}) \gamma^{\mu} b\right| \bar{B}_{(s)}\left(P_{B_{(s)}}\right)\right\rangle= & -\frac{2 V\left(q^{2}\right)}{m_{B_{(s)}}+m_{T}} \epsilon^{\mu \nu \rho \sigma} \epsilon_{T_{\nu}}^{*} P_{B_{s} \rho} P_{T \sigma}, \\
\left\langle T\left(P_{T}, \epsilon\right)\left|\bar{s} \gamma^{\mu} \gamma_{5} b\right| \bar{B}_{(s)}\left(P_{B_{(s)}}\right)\right\rangle= & 2 i m_{T} A_{0}\left(q^{2}\right) \frac{\epsilon_{T}^{*} \ldots q}{q^{2}} q^{\mu}+i\left(m_{B_{(s)}}+m_{T}\right) A_{1}\left(q^{2}\right)\left[\epsilon_{T_{\mu}}^{*}-\frac{\epsilon_{T}^{*} \ldots q}{q^{2}} q^{\mu}\right] \\
& -i A_{2}\left(q^{2}\right) \frac{\epsilon_{T}^{*} \ldots q}{m_{B_{(s)}}+m_{f_{2}^{\prime}}}\left[P^{\mu}-\frac{m_{B_{(s)}}^{2}+m_{T}^{2}}{q^{2}} q^{\mu}\right], \\
\left\langle T\left(P_{T}, \epsilon\right)\left|\bar{s} \sigma^{\mu \nu} q_{\nu} b\right| \bar{B}_{(s)}\left(P_{B_{(s)}}\right)\right\rangle= & -2 i T_{1}\left(q^{2}\right) \epsilon^{\mu \nu \rho \sigma} \epsilon_{T_{\nu}}^{*} P_{B_{(s)} \rho} P_{T \sigma}, \\
\left\langle T\left(P_{T}, \epsilon\right)\left|(\bar{s}) \sigma^{\mu \nu} \gamma_{5} q_{\nu} b\right| \bar{B}_{(s)}\left(P_{B_{(s)}}\right)\right\rangle= & T_{2}\left(q^{2}\right)\left[\left(m_{B_{(s)}}^{2}+m_{T}^{2}\right) \epsilon_{T_{\mu}} \epsilon_{T}^{*} \ldots q P^{\mu}\right]+T_{3}\left(q^{2}\right) \epsilon_{T}^{*} \ldots q\left[q^{\mu}-\frac{q^{2}}{m_{B_{(s)}}^{2}+m_{T}^{2}} P^{\mu}\right],
\end{aligned}
$$

where the momentum transfer $q=P_{B_{(s)}}-P_{T}$. We use the relevant form factors in our analysis for $B_{(s)}$ to light $J^{P C}=$ $2^{++}$tensor meson (T) derived from the LCSR approach. The parameterized $q^{2}$-dependent form factors are given in the form as [32]

$$
F^{B_{(s)} T}\left(q^{2}\right)=\frac{F^{B_{(s)} T}(0)}{1-a_{T}\left(q^{2} / m_{B_{q}}^{2}\right)+b_{T}\left(q^{2} / m_{B_{q}}^{2}\right)^{2}},
$$

where $F=V, A_{0}, A_{1}, A_{2}, T_{1}, T_{2}$, and $T_{3}$. The symbol $T$ denotes the tensor mesons $K_{2}^{*}(1430)$ and $f_{2}^{\prime}(1525)$.

\section{FORMULAS OF BRANCHING RATIO AND OTHER OBSERVABLES}

The transition amplitude for $B \rightarrow K_{2}^{*}(1430) \ell^{+} \ell^{-}$and $B_{s} \rightarrow f_{2}^{\prime}(1525) \ell^{+} \ell^{-}$processes can be obtained from the generalized effective Hamiltonian given in Eq. (2). The $q^{2}$-dependent differential decay rate for the semileptonic $B_{(s)} \rightarrow T \ell^{+} \ell^{-}\left(T=f_{2}^{\prime}, K_{2}^{*}\right)$ modes mediated by $b \rightarrow s \ell^{+} \ell^{-}$parton level can be given as [26,27,52]

$$
\frac{d \Gamma}{d q^{2}}=\frac{1}{4}\left(3 I_{1}^{c}+6 I_{1}^{s}-I_{2}^{c}-2 I_{2}^{s}\right),
$$

where the angular coefficients $I_{i}\left(q^{2}\right)$ are defined as 


$$
\begin{aligned}
& I_{1}^{c}=\left(\left|A_{L 0}\right|^{2}+\left|A_{R 0}\right|^{2}\right)+8 \frac{m_{\ell}^{2}}{q^{2}} \operatorname{Re}\left[A_{L 0} A_{R 0}^{*}\right]+4 \frac{m_{\ell}^{2}}{q^{2}}\left|A_{t}\right|^{2}, \\
& I_{1}^{s}=\frac{3}{4}\left[\left|A_{L \perp}\right|^{2}+\left|A_{L \|}\right|^{2}+\left|A_{R \perp}\right|^{2}+\left|A_{R \|}\right|^{2}\right]\left(1-\frac{4 m_{\ell}^{2}}{3 q^{2}}\right)+\frac{4 m_{\ell}^{2}}{q^{2}} \operatorname{Re}\left[A_{L \perp} A_{R \perp}^{*}+A_{L \|} A_{R \|}^{*}\right], \\
& I_{2}^{c}=-\left(1-\frac{4 m_{\ell}^{2}}{q^{2}}\right)\left(\left|A_{L 0}\right|^{2}+\left|A_{R 0}\right|^{2}\right), \\
& I_{2}^{s}=\frac{1}{4}\left(1-\frac{4 m_{\ell}^{2}}{q^{2}}\right)\left[\left|A_{L \perp}\right|^{2}+\left|A_{L \|}\right|^{2}+\left|A_{R \perp}\right|^{2}+\left|A_{R \|}\right|^{2}\right] .
\end{aligned}
$$

The explicit expressions of the transversity amplitudes given in the above equation can be written as follows:

$$
\begin{aligned}
& A_{L 0}=N_{T} \frac{\sqrt{\lambda\left(m_{(s)}^{2}, m_{T}^{2}, q^{2}\right)}}{\sqrt{6} m_{B_{(s)}} m_{T}} \frac{1}{2 m_{T} \sqrt{q^{2}}}\left\{\left(C_{9}^{\mathrm{eff}}-C_{10}\right)\left[\left(m_{B_{(s)}}^{2}-m_{T}^{2}-q^{2}\right)\left(m_{B_{(s)}}+m_{T}\right) A_{1}-\frac{\lambda\left(m_{(s)}^{2}, m_{T}^{2}, q^{2}\right)}{m_{B_{(s)}}+m_{T}} A_{2}\right]\right. \\
& \left.+2 m_{b} C_{7}^{\mathrm{eff}}\left[\left(m_{B_{(s)}}^{2}+3 m_{T}^{2}-q^{2}\right) T_{2}-\frac{\lambda\left(m_{(s)}^{2}, m_{T}^{2}, q^{2}\right)}{m_{B_{(s)}}^{2}-m_{T}^{2}} T_{3}\right]\right\} \\
& A_{L \perp}=-N_{T} \sqrt{2} \frac{\sqrt{\lambda\left(m_{(s)}^{2}, m_{T}^{2}, q^{2}\right)}}{\sqrt{8} m_{B_{(s)}} m_{T}}\left[\left(C_{9}^{\mathrm{eff}}-C_{10}\right) \frac{\sqrt{\lambda\left(m_{(s)}^{2}, m_{T}^{2}, q^{2}\right)}}{m_{B_{(s)}}+m_{T}} V+\frac{\sqrt{\lambda\left(m_{(s)}^{2}, m_{T}^{2}, q^{2}\right)} 2 m_{b} C_{7}^{\mathrm{eff}}}{q^{2}} T_{1}\right], \\
& A_{L \|}=N_{T} \sqrt{2} \frac{\sqrt{\lambda\left(m_{(s)}^{2}, m_{T}^{2}, q^{2}\right)}}{\sqrt{8} m_{B_{(s)}} m_{T}}\left[\left(C_{9}^{\mathrm{eff}}-C_{10}\right)\left(m_{B_{(s)}}+m_{T}\right) A_{1}+\frac{2 m_{b} C_{7}^{\mathrm{eff}}\left(m_{B_{(s)}}^{2}-m_{T}^{2}\right)}{q^{2}} T_{2}\right], \\
& A_{t}=2 N_{T} \frac{\sqrt{\lambda\left(m_{(s)}^{2}, m_{T}^{2}, q^{2}\right)}}{\sqrt{6} m_{B_{(s)}} m_{T}} C_{10} \frac{\sqrt{\lambda\left(m_{(s)}^{2}, m_{T}^{2}, q^{2}\right)}}{\sqrt{q^{2}}} A_{0}, \\
& A_{R i}=\left.A_{L i}\right|_{C_{10} \rightarrow-C_{10}}, \quad(i=0, \perp, \|),
\end{aligned}
$$

where the normalization factor is given as

$$
\begin{aligned}
N_{T}= & {\left[\frac{G_{F}^{2} \alpha^{2}}{3 \cdot 2^{10} \pi^{5} m_{B_{(s)}^{3}}^{3}}\left|V_{t b} V_{t s}^{*}\right|^{2} q^{2} \sqrt{\lambda\left(m_{(s)}^{2}, m_{T}^{2}, q^{2}\right)}\right.} \\
& \left.\times\left(1-\frac{4 m_{l}^{2}}{q^{2}}\right)^{1 / 2}\right]^{1 / 2}
\end{aligned}
$$

and the parameter $\lambda$ is defined in Eq. (10). Now, in order to scrutinize the structure of new physics, we explore with various interesting observables for the processes $B_{(s)} \rightarrow T \ell^{+} \ell^{-}$given as follows [52]:

(i) differential branching ratio

$$
\mathcal{B R}\left(q^{2}\right)=\tau_{B_{(s)}} \frac{d \Gamma}{d q^{2}}=\tau_{B_{(s)}} \frac{1}{4}\left(3 I_{1}^{c}+6 I_{1}^{s}-I_{2}^{c}-2 I_{2}^{s}\right)
$$

(ii) forward-backward asymmetry

$$
\begin{aligned}
\left\langle\mathcal{A}_{\mathcal{F B}}\right\rangle & =\frac{\left(\int_{0}^{1}-\int_{-1}^{0}\right) d \cos \theta \frac{d^{2} \Gamma}{d q^{2} d \cos \theta}}{d \Gamma / d q^{2}} \\
& =\frac{3 I_{6}}{3 I_{1}^{c}+6 I_{1}^{s}-I_{2}^{c}-2 I_{2}^{s}},
\end{aligned}
$$

where

$$
I_{6}=2 \sqrt{1-4 m_{\ell}^{2} / q^{2}}\left[\operatorname{Re}\left(A_{L \|} A_{L \perp}^{*}\right)-\operatorname{Re}\left(A_{R \|} A_{R \perp}^{*}\right)\right]
$$

(iii) longitudinal polarization fraction

$$
\left\langle\mathcal{F}_{\mathcal{L}}\right\rangle=\frac{\int_{q_{\text {low }}^{2}}^{q_{\text {ligh }}^{2}} d q^{2} \frac{d \Gamma_{L}}{d q^{2}}}{\int_{q_{\text {low }}^{2}}^{q_{\text {high }}^{2}} d q^{2} \frac{d \Gamma}{d q^{2}}}=\frac{3 I_{1}^{c}-I_{2}^{c}}{3 I_{1}^{c}+6 I_{1}^{s}-I_{2}^{c}-2 I_{2}^{s}} ;
$$


(iv) angular observable

$$
\left\langle\mathcal{P}_{5}^{\prime}\right\rangle=\frac{\int_{q_{\text {low }}^{2}}^{q_{\text {high }}^{2}} I_{5}}{2 \sqrt{-\int_{q_{\text {low }}^{2}}^{q_{\text {high }}^{2}} d q^{2} I_{2}^{c} \int_{q_{\text {low }}^{2}}^{q_{\text {high }}^{2}} d q^{2} I_{2}^{s}}} .
$$

However, there are several other observables that can also be constructed and are very sensitive to the window of NP. These are defined in the form of ratios and differences between the observables associated with two different lepton families and are given explicitly as follows:

(i) lepton flavor universality violation parameter

$$
\mathcal{R}_{e}^{\mu}\left(q_{\text {low }}^{2}, q_{\text {high }}^{2}\right)=\frac{\int_{q_{\text {liow }}^{2}}^{q_{\text {high }}^{2}} d q^{2} d \mathcal{B} \mathcal{R}_{\mu} / d q^{2}}{\int_{q_{\text {low }}^{\text {ligh }}}^{q_{\text {lig }}^{2}} d q^{2} d \mathcal{B} \mathcal{R}_{e} / d q^{2}}
$$

(ii) the $\left\langle Q_{i}\right\rangle\left(i=\mathcal{F}_{\mathcal{L}}, \mathcal{A}_{\mathcal{F} \mathcal{B}}, Q_{5}^{\prime}\right)$ parameter

$$
\begin{aligned}
\left\langle Q_{\mathcal{F}_{\mathcal{L}}}\right\rangle & =\left\langle\mathcal{F}_{\mathcal{L}}^{\mu}\right\rangle-\left\langle\mathcal{F}_{\mathcal{L}}^{e}\right\rangle, \quad\left\langle Q_{\mathcal{A}_{\mathcal{F B}}}\right\rangle=\left\langle\mathcal{A}_{\mathcal{F B}}^{\mu}\right\rangle-\left\langle\mathcal{A}_{\mathcal{F} \mathcal{B}}^{e}\right\rangle, \\
\left\langle Q_{5}^{\prime}\right\rangle & =\left\langle Q_{5}^{\mu}\right\rangle-\left\langle Q_{5}^{e}\right\rangle .
\end{aligned}
$$

\section{NEW PHYSICS ANALYSIS}

A heavy $Z^{\prime}$ boson, in the tree-level exchange with flavor changing neutral current transition mediated by $b \rightarrow$ $s \ell^{+} \ell^{-}$parton level, is the most obvious candidate in the NP contribution. There are different scenarios which are responsible for muonic four-fermion $b \rightarrow s \mu^{+} \mu^{-}$NP operators and are given as follows:

$$
\begin{gathered}
\text { (I) }:\left[\bar{s}_{\mu} P_{L} b\right]\left[\bar{\mu} \gamma^{\mu} \mu\right], \\
(\mathrm{II}):\left[\bar{s} \gamma_{\mu} P_{L} b\right]\left[\bar{\mu} \gamma^{\mu} P_{L} \mu\right], \\
(\mathrm{III}):\left[\bar{s} \gamma_{\mu} \gamma_{5} b\right]\left[\bar{\mu} \gamma^{\mu} \mu\right] .
\end{gathered}
$$

However, scenarios (I) and (II) display the $Z^{\prime}$ boson to couple with the quark sector $\bar{s}_{L}-b_{L}-Z^{\prime}$ and the lepton sector $Z^{\prime}-\bar{\mu}-\mu$ vectorially, whereas it couples axialvectorially in scenario (III). Having said that, we exclude scenario (III), as it is strongly rejected by the $R_{K}$ measurement. The $Z^{\prime}$ boson must transform as a singlet or triplet under $S U(2)_{L}$ gauge group as it couples to left-handed quarks. In the case of a triplet [53-55], a new gauge boson $W^{\prime}$ can contribute to $B \rightarrow D^{(*)+} \tau^{-} \bar{\nu}_{\tau}$ mediated by $b \rightarrow c$ quark-level transition, where the deviation in the measurement has been observed in Refs. [56,57]. In the case of a singlet under $S U(2)_{L}$ gauge group, this $Z^{\prime}$ gauge boson is associated with an extension of Abelian $U(1)^{\prime}$ group to the SM. Many works have been proposed in this model with the scenario $C_{9}^{\mu \mu}(N P)=-C_{10}^{\mu \mu}(N P)$ [58-63], where the Wilson coefficients are $q^{2}$ independent. However, on the other hand, it is very interesting to consider a light $Z^{\prime}$ which can also address $b \rightarrow s \mu^{+} \mu^{-}$data $[33,43,44]$. If $2 m_{\mu}<m_{Z^{\prime}}<m_{B}$, a resonance state can be obtained in the dimuon invariant mass. Moreover to say that, since no signature for such a kind of state has been observed in the dimuon invariant mass, we consider the typical $Z^{\prime}$ mass less than $2 m_{\mu}$, i.e., $200 \mathrm{MeV}$ in our analysis. For the coupling $\bar{s} b$ with the light $Z^{\prime}$, the general form of the flavor changing vertex $\bar{s} b Z^{\prime}$ is considered as [33]

$$
F\left(q^{2}\right) \bar{s} \gamma^{\mu} P_{L} b Z_{\mu}^{\prime},
$$

where the form of the form factor $F\left(q^{2}\right)$ can be written as

$$
F\left(q^{2}\right)=a_{L}^{b s}+g_{L}^{b s} \frac{q^{2}}{m_{B}^{2}}+\cdots
$$

The leading-order term $a_{L}^{b s}$ given in the above equation is severely constrained by $B \rightarrow K \nu \bar{\nu}$ and can be neglected, and we consider the coupling $g_{L}^{b s}$ only. Thus, the $q^{2}$-dependent NP Wilson coefficients for $b \rightarrow s \mu^{+} \mu^{-}$transition are given as

$$
\begin{aligned}
& C_{9}^{\mu \mu}(N P)=\mathcal{G} \frac{g_{b s}^{L} q^{2} / m_{B}^{2}\left(g_{\mu \mu}^{L}+g_{\mu \mu}^{R}\right)}{q^{2}-m_{Z^{\prime}}^{2}}, \\
& C_{10}^{\mu \mu}(N P)=-\mathcal{G} \frac{g_{b s}^{L} q^{2} / m_{B}^{2}\left(g_{\mu \mu}^{L}-g_{\mu \mu}^{R}\right)}{q^{2}-m_{Z^{\prime}}^{2}},
\end{aligned}
$$

where $\mathcal{G}=\frac{\pi}{\sqrt{2} G_{F} \alpha V_{t b} V_{t s}^{*}}$. It has been pointed out in Ref. [33] that one can explain the B anomalies as good as in the case of a heavy $Z^{\prime}$ boson. It is clearly reported that, except $R_{K^{*}}$ measurement in the low $q^{2}$ bin range, the light $Z^{\prime}$ with pure vector coupling to muon can easily accommodate the clean observables $R_{K}^{[1,6]}$ and $R_{K^{*}}^{[1,1,6]}$ data given in Table I in Ref. [43]. Since we assume the NP to exist in the muonic mode of $b \rightarrow s \ell^{+} \ell^{-}$transition, the NP coupling $C_{9}^{\mu \mu}(N P)$ is considered in our analysis where the light $Z^{\prime}$ couples with the muon vectorially under the condition $g_{\mu \mu}^{L}=g_{\mu \mu}^{R}=g_{\mu \mu}$.

The long-standing discrepancy between theory and experiment that concerns the anomalous magnetic dipole moment of the muon, i.e., $a_{\mu}=(g-2) / 2$, has caused

TABLE I. Wilson coefficients $C_{i}\left(m_{b}\right)$ in the leading logarithmic approximation [70].

\begin{tabular}{lcccccccc}
\hline \hline$C_{1}$ & $C_{2}$ & $C_{3}$ & $C_{4}$ & $C_{5}$ & $C_{6}$ & $C_{7}^{\text {eff }}$ & $C_{9}$ & $C_{10}$ \\
\hline-0.248 & 1.107 & 0.011 & -0.026 & 0.007 & -0.031 & -0.313 & 4.344 & -4.669 \\
\hline \hline
\end{tabular}


excitement among theorists. The combination of the recent updates on the measurements from Fermilab [64] and the previous result obtained from Brookhaven National Laboratory E82 [65] leads to a new average value with $4.2 \sigma$ deviation from the SM result [66] and is given as follows:

$$
\begin{aligned}
& a_{\mu}^{\mathrm{SM}}=116591810(43) \times 10^{-11}, \\
& a_{\mu}^{\exp }=116592061(43) \times 10^{-11}, \\
& \Delta a_{\mu} \equiv a_{\mu}^{\exp }-a_{\mu}^{\mathrm{SM}}=(2.51 \pm 0.59) \times 10^{-9} .
\end{aligned}
$$

As the light $Z^{\prime}$ can also explain the muon $(g-2)$ anomaly, from Ref. [67] the expression of the absolute magnitude of the discrepancy $\Delta a_{\mu}$ is given as

$$
\Delta a_{\mu}=\frac{\left(g_{\mu \mu}\right)^{2}}{8 \pi^{2}} \int_{0}^{1} \frac{2 x^{2}(1-x)}{x^{2}+\left(m_{Z^{\prime}}^{2} / m_{\mu}^{2}\right)(1-x)} d x
$$

where $m_{Z^{\prime}}$ is the mass of the light $Z^{\prime}$ boson, $m_{\mu}$ is the mass of the muon, and the coupling $g_{\mu \mu}=1.42 \times 10^{-3}$ is obtained for $m_{Z^{\prime}}=200 \mathrm{MeV}$.

\section{RESULTS AND DISCUSSION}

\section{A. Relevant input parameters}

In this subsection, we report all the relevant inputs used for the numerical calculations of the various decay observables. In our analysis, the input parameters such as mean lifetime and masses of $B_{(s)}$, the tensor mesons and lepton masses, and the Fermi coupling constant are given as follows [68]:

$$
\begin{aligned}
\tau_{B} & =1.638 \times 10^{-12} \mathrm{sec}, \quad m_{B}=5.27934 \mathrm{GeV}, \quad m_{B_{s}}=5.36688 \mathrm{GeV}, \\
\tau_{B_{s}} & =1.515 \times 10^{-12} \mathrm{sec}, \quad m_{K_{2}^{*}}=1.430 \mathrm{GeV}, \quad m_{f_{2}^{\prime}}=1.525 \mathrm{GeV}, \\
G_{F} & =1.1663787 \times 10^{-5} \mathrm{GeV}^{-2}, \quad m_{e}=0.5109989461 \times 10^{-3} \mathrm{GeV}, \quad m_{\mu}=0.1056583715 \mathrm{GeV} .
\end{aligned}
$$

Similarly for the quark masses, we use $m_{b}^{\text {pole }}=4.8$ $\mathrm{GeV}, m_{b}^{(\overline{\mathrm{MS}})}=4.2 \mathrm{GeV}$, and $m_{c}^{(\overline{\mathrm{MS}})}=1.28 \mathrm{GeV}$ [69]. From Ref. [68], we also consider the fine structure constant $\alpha=$ $1 / 133.28$ and the CKM parameter $\left|V_{t b} V_{t s}\right|=0.04088(55)$. The inputs of the Wilson coefficients in the leading logarithm approximation calculated at $\mu=4.8$ are taken from Ref. [70] and are given in Table I.

However, we report the relevant form factors required for the computation of the decay observables from Ref. [32]. The explicit entries of the form factors at $q^{2}=0$ with the fitted parameters $a$ and $b$ are given in Table II.

\section{B. $\chi^{2}$ analysis}

To obtain the discrepancy of the SM with the experimental data, we perform a naive $\chi^{2}$ analysis with the existing $b \rightarrow s \ell \ell$ data. In our fit, we include only the updated experimental result obtained from $\mathrm{LHCb}$ for

TABLE II. The relevant form factors with the fitted parameters [32].

\begin{tabular}{lcc}
\hline \hline & {$\left[F^{B K_{2}^{*}}(0), a_{K_{2}^{*}}, b_{K_{2}^{*}}\right]$} & {$\left[F^{B_{s} f_{2}^{\prime}}(0), a_{f_{2}^{\prime}}, b_{f_{2}^{\prime}}\right]$} \\
\hline$V$ & {$[0.16 \pm 0.02,2.08,1.50]$} & {$[0.15 \pm 0.02,2.06,1.49]$} \\
$A_{0}$ & {$[0.25 \pm 0.04,1.57,0.10]$} & {$[0.25 \pm 0.04,1.72,0.31]$} \\
$A_{1}$ & {$[0.14 \pm 0.02,1.23,0.49]$} & {$[0.13 \pm 0.02,1.25,0.47]$} \\
$A_{2}$ & {$[0.05 \pm 0.02,1.32,14.9]$} & {$[0.03 \pm 0.02,4.71,105]$} \\
$T_{1}$ & {$[0.14 \pm 0.02,2.07,1.50]$} & {$[0.13 \pm 0.02,2.06,1.49]$} \\
$T_{2}$ & {$[0.14 \pm 0.02,1.22,0.35]$} & {$[0.13 \pm 0.02,1.23,0.32]$} \\
$T_{3}$ & {$\left[0.01_{-0.01}^{+0.02}, 9.91,276\right]$} & {$\left[0.00_{-0.01}^{+0.02},-,-\right]$} \\
\hline \hline
\end{tabular}

$R_{K}^{[1.1,6.0]}$ [7] and $R_{K^{*}}^{[1,1,6.0]}$ [2] in our analysis, as the $R_{K^{*}}$ measurement in the bin range $0.045 \leq q^{2} \leq 1.1 \mathrm{GeV}^{2}$ does not accommodate within $1 \sigma$ deviation. The $\chi^{2}$ is defined as

$$
\chi^{2}=\sum_{i} \frac{\left(\mathcal{O}_{i}^{\mathrm{th}}\left(C_{9}^{\mathrm{NP}}\right)-\mathcal{O}_{i}^{\operatorname{Exp}}\right)^{2}}{\left(\Delta \mathcal{O}_{i}^{2}\right)}
$$

where the numerator includes the theoretical contributions $\mathcal{O}_{i}^{\text {th }}$ with the NP coupling and the measured central values $\mathcal{O}_{i}^{\text {Exp }}$. of the observables and $\Delta \mathcal{O}_{i}^{2}=\left(\Delta \mathcal{O}_{i}^{\text {Exp }}\right)^{2}+\left(\Delta \mathcal{O}_{i}^{\text {SM }}\right)^{2}$. The denominator envelopes $1 \sigma$ uncertainties from theory and experimental results. Considering the coupling as real, we obtain the best-fit value of the NP coupling associated with the $Z^{\prime}$ boson as $g_{b s}^{L}=1.57 \times 10^{-5}$.

$$
\begin{gathered}
\text { C. } B \rightarrow K_{2}^{*}(1430) \ell^{+} \ell^{-} \text {and } B_{s} \rightarrow f_{2}^{\prime}(1525) \ell^{+} \ell^{-} \\
\text {decay observables }
\end{gathered}
$$

\section{Analysis of $B_{s} \rightarrow f_{2}^{\prime}(1525) \ell^{+} \ell^{-}$ in the $S M$ and beyond}

We analyze the rare exclusive $B_{(s)} \rightarrow T \ell^{+} \ell^{-}$ $\left(T=f_{2}^{\prime}, K_{2}^{*}\right)$ processes in the presence of the light $Z^{\prime}$ model where the coupling arises from only the $C_{9}^{N P}$ contribution; in other words, the coupling corresponds to the vectorial contribution to the muon. Using the NP coupling, we report the impact on various observables such as differential branching ratio, the lepton polarization fraction $F_{L}$, the forward-backward asymmetry $A_{F B}$, and the angular observable $P_{5}^{\prime}$. Additionally, some other important 
LFU-sensitive observables such as $R_{T}\left(T=f_{2}^{\prime}, K_{2}^{*}\right), Q_{F_{L}}$, $Q_{A_{F B}}$, and $Q_{5}^{\prime}$ are also investigated in this analysis. With all the input parameters that are pertinent to our analysis, we display the variations of all the observables with respect to $q^{2}$ in Fig. 1. Similarly, in Fig. 2, we show the corresponding $q^{2}$ binwise plots for $B_{s} \rightarrow f_{2}^{\prime} \ell \ell$ decay mode where we choose different bin sizes such as [0.1, 0.98], [1.1, 2.5], [2.5, 4], [4.0, 6.0], and $[1.1,6]$ (in units of $\mathrm{GeV}^{2}$ ) compatible with $\mathrm{LHCb}$ measurements. The binwise predictions along with its $1 \sigma$ standard deviation both in SM and in the presence of the $Z^{\prime}$ model in several $q^{2}$ bin rooms have been reported in
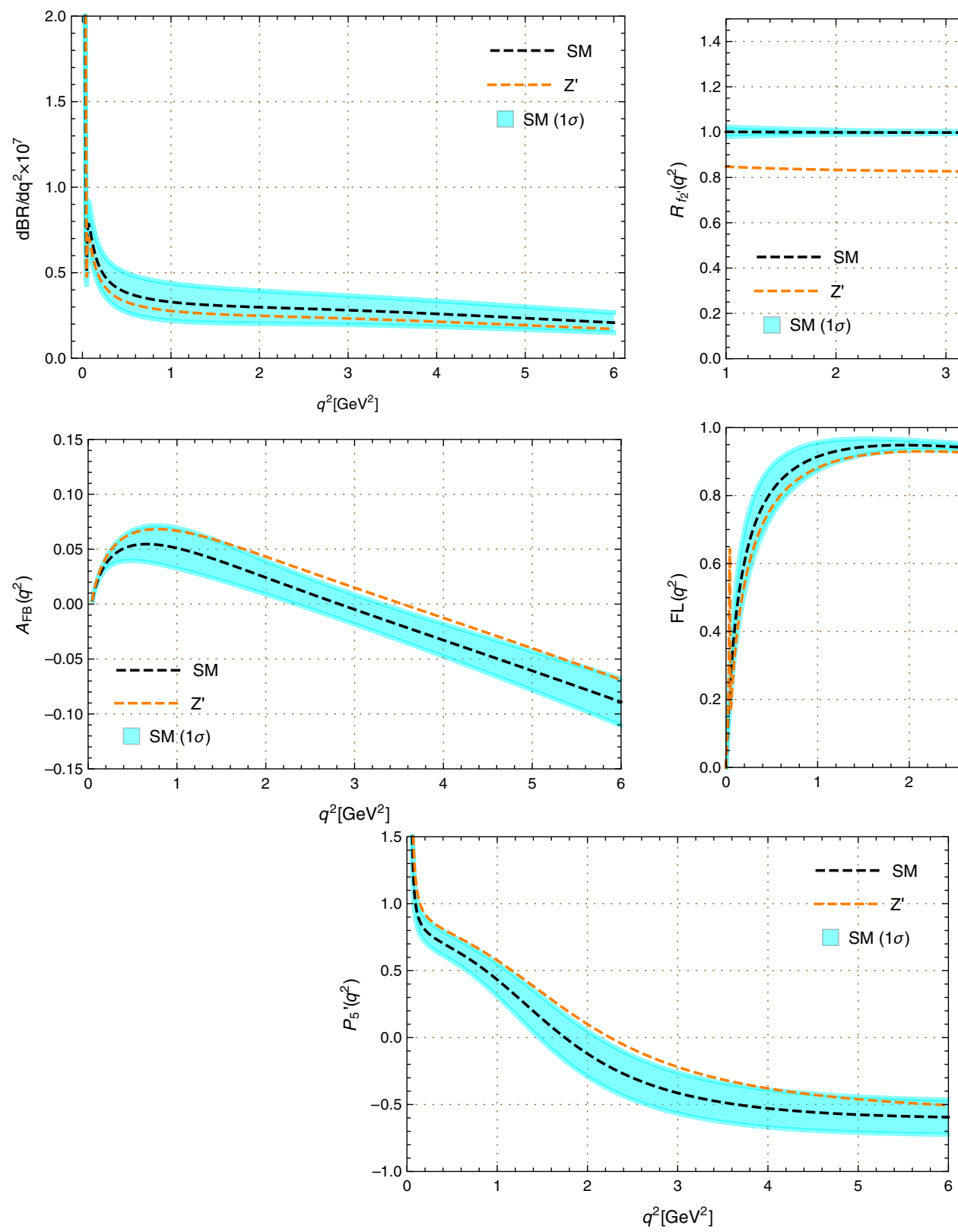

Table III. We provide our detailed observations in the presence of the NP contribution as below.

Description of the color inputs for the following plots:

Distribution plot.-Black dotted line, SM contribution; cyan band, $1 \sigma$ error band due to form factors and CKM element; orange dotted line, light $Z^{\prime}$ contribution.

Binwise plot.-Black bins, SM central values; yellow band, $1 \sigma$ uncertainty due to form factors and CKM element; green bin, light $Z^{\prime}$ contribution.

(i) Branching ratio $(B R)$.- - In the top-left panel in Fig. 1, we show the $q^{2}$ dependency of the branching
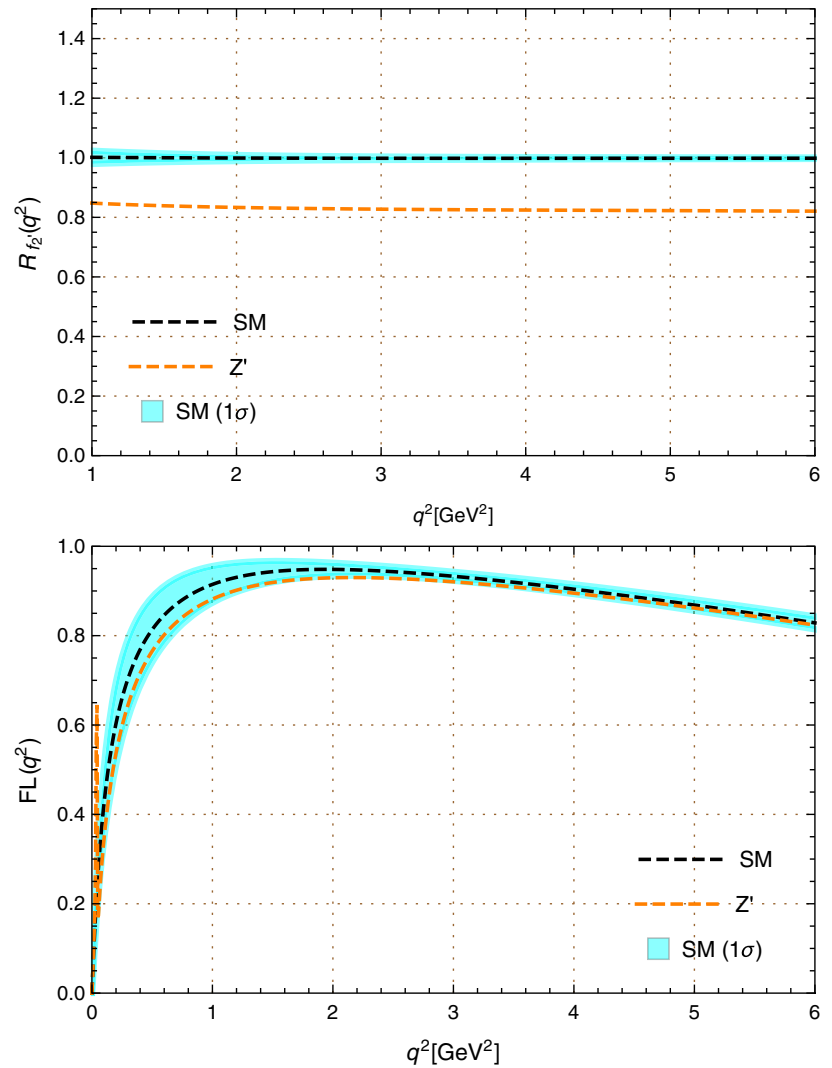

FIG. 1. The $q^{2}$ distribution of various observables such as branching ratio, the polarization fraction, the forward-backward asymmetry, and $P_{5}^{\prime}$ for the $B_{s} \rightarrow f_{2}^{\prime}(1525) \ell^{+} \ell^{-}$process (black dotted line, SM contribution; cyan band, $1 \sigma$ uncertainty due to form factors and CKM element; orange dotted line, $Z^{\prime}$ contribution). 

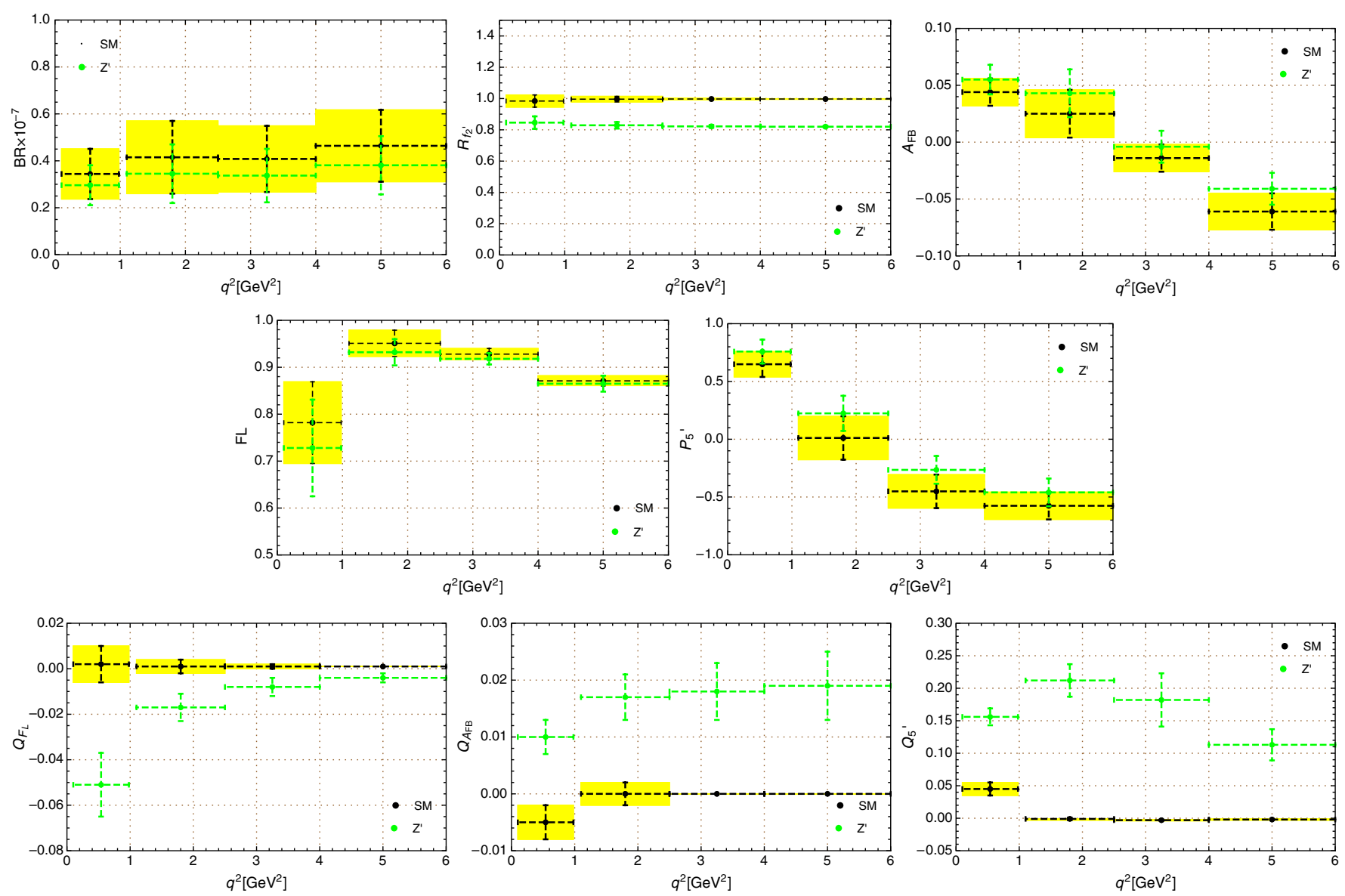

FIG. 2. The binwise distributions of observables such as branching ratio, the polarization fraction, the forward-backward asymmetry, $P_{5}^{\prime}$, and the sensitive LFU parameters $R_{f_{2}^{\prime}}, Q_{F_{L}}, Q_{A_{F B}}$, and $Q_{5}^{\prime}$ of $B_{s} \rightarrow f_{2}^{\prime} \ell^{+} \ell^{-}$processes (black bins, SM central values; yellow band, $1 \sigma$ uncertainty due to form factors and CKM element; green bin, $Z^{\prime}$ contribution).

TABLE III. Prediction of various observables with $1 \sigma$ standard deviation in the SM and $Z^{\prime}$ model for the $B_{s} \rightarrow f_{2}^{\prime} \ell^{+} \ell^{-}$process in different bin rooms.

\begin{tabular}{lcccccc}
\hline \hline Observable & & {$[0.10,0.98]$} & {$[1.1,2.5]$} & {$[2.5,4.0]$} & {$[4.0,6.0]$} & {$[1.1,6.0]$} \\
\hline \multicolumn{7}{c}{$B_{s} \rightarrow f_{2}^{\prime} \mu^{+} \mu^{-}$} \\
\hline $\mathcal{B R} \times 10^{-7}$ & $S M$ & $0.344 \pm 0.107$ & $0.415 \pm 0.155$ & $0.408 \pm 0.141$ & $0.464 \pm 0.153$ & $1.287 \pm 0.449$ \\
& $Z^{\prime}$ & $0.296 \pm 0.085$ & $0.345 \pm 0.125$ & $0.337 \pm 0.114$ & $0.381 \pm 0.124$ & $1.064 \pm 0.364$ \\
$\mathcal{A}_{\mathcal{F B}}$ & $S M$ & $0.044 \pm 0.012$ & $0.025 \pm 0.021$ & $-0.014 \pm 0.012$ & $-0.061 \pm 0.016$ & $-0.018 \pm 0.014$ \\
& $Z^{\prime}$ & $0.055 \pm 0.013$ & $0.043 \pm 0.021$ & $0.004 \pm 0.014$ & $-0.041 \pm 0.014$ & $0.000 \pm 0.013$ \\
$\mathcal{F}_{\mathcal{L}}$ & $S M$ & $0.782 \pm 0.087$ & $0.951 \pm 0.028$ & $0.928 \pm 0.012$ & $0.871 \pm 0.011$ & $0.915 \pm 0.013$ \\
& $Z^{\prime}$ & $0.728 \pm 0.103$ & $0.932 \pm 0.028$ & $0.918 \pm 0.012$ & $0.865 \pm 0.017$ & $0.903 \pm 0.013$ \\
$\mathcal{P}_{5}^{\prime}$ & $S M$ & $0.649 \pm 0.110$ & $0.011 \pm 0.188$ & $-0.451 \pm 0.145$ & $-0.576 \pm 0.118$ & $-0.381 \pm 0.140$ \\
& $Z^{\prime}$ & $0.759 \pm 0.103$ & $0.224 \pm 0.152$ & $-0.265 \pm 0.140$ & $-0.460 \pm 0.120$ & $-0.209 \pm 0.149$ \\
$\mathcal{R}_{e}^{\mu}$ & $S M$ & $0.984 \pm 0.039$ & $0.996 \pm 0.018$ & $0.997 \pm 0.005$ & $0.997 \pm 0.002$ & $0.997 \pm 0.006$ \\
& $Z^{\prime}$ & $0.846 \pm 0.060$ & $0.829 \pm 0.021$ & $0.822 \pm 0.009$ & $0.820 \pm 0.006$ & $0.823 \pm 0.010$ \\
$Q_{\mathcal{A}_{\mathcal{F B}}}$ & $S M$ & $-0.005 \pm 0.003$ & $-0.000 \pm 0.002$ & $0.000 \pm 0.000$ & $0.000 \pm 0.000$ & $-0.000 \pm 0.000$ \\
$Q_{\mathcal{F}_{\mathcal{L}}}$ & $Z^{\prime}$ & $0.010 \pm 0.003$ & $0.017 \pm 0.004$ & $0.018 \pm 0.005$ & $0.019 \pm 0.006$ & $0.018 \pm 0.004$ \\
$Q_{5}^{\prime}$ & $S M$ & $0.002 \pm 0.008$ & $0.001 \pm 0.003$ & $0.001 \pm 0.001$ & $0.001 \pm 0.000$ & $0.001 \pm 0.000$ \\
& $Z^{\prime}$ & $-0.051 \pm 0.014$ & $-0.017 \pm 0.006$ & $-0.008 \pm 0.004$ & $-0.004 \pm 0.002$ & $-0.010 \pm 0.003$ \\
\hline \hline & $S M$ & $0.045 \pm 0.010$ & $-0.001 \pm 0.002$ & $-0.003 \pm 0.001$ & $-0.002 \pm 0.001$ & $-0.004 \pm 0.001$ \\
\hline
\end{tabular}


ratio for $B_{s} \rightarrow f_{2}^{\prime} \ell^{+} \ell^{-}$decay within the $\mathrm{SM}$ as well as in the presence of the light $Z^{\prime}$ model for the $\mu$ mode. We observe that the $q^{2}$ behavior of the observable in the presence of light $Z^{\prime}$ is reduced and lies within the SM $1 \sigma$ uncertainty band. Similarly, we proceed with the binwise plot of the branching ratio in the top-left panel in Fig. 2. However, though the numerical values in the presence of light $Z^{\prime}$ differ from the SM contribution, no such remarkable deviations are observed in this analysis.

(ii) Forward-backward asymmetry $\left(A_{F B}\right)$.-We display the $q^{2}$ variation of forward-backward asymmetry in the middle-left panel in Fig. 1. In the presence of the light $Z^{\prime}$ contribution, its $q^{2}$ behavior shifted to higher values as compared to $\mathrm{SM}$ variations in all bin rooms. In the SM variation, the observable $A_{F B}\left(q^{2}\right)$ has zero crossing at $\sim 2.8 \mathrm{GeV}^{2}$, whereas the crossing point shifted to $\sim 3.5 \mathrm{GeV}^{2}$ in the presence of new physics. Again, we observe that, in all bins given in Table III, the NP contributions lie within $1 \sigma$ from the SM predictions.

(iii) Longitudinal polarization fraction $\left(F_{L}\right)$. - From the $q^{2}$ distribution plot given in the middle-right panel in Fig. 1, one can observe that due to the NP coupling the contribution shifted lower to the SM values in all $q^{2}$ bins. However, we do not draw any significant deviations for this observable.

(iv) The angular observable $\left(P_{5}^{\prime}\right)$.- - For the angular observable $P_{5}^{\prime}$ given in the bottom panel in Fig. 1, in the presence of NP coupling this observable is clearly distinguished from the SM contributions. However, we observe that the NP coupling shifts the contribution to higher values as compared to the SM. The zero crossing occurs at nearly $\sim 1.8 \mathrm{GeV}^{2}$ for the SM, whereas in presence of NP coupling it touches at $\sim 2.3 \mathrm{GeV}^{2}$ for the same. This observable becomes negative in the $q^{2}$ regions $[2.5,4],[4,6]$, and $[1.1,6]$, whereas it remains positive in other bin ranges.

(v) LFU-sensitive parameter $\left(R_{f_{2}^{\prime}}\right)$.- Interestingly, the ratio of the branching ratio (in other words, the LFU-sensitive parameter $R_{f_{2}^{\prime}}$ ) is clearly distinguishable from the SM prediction $(\simeq 1)$ with more than $5 \sigma$ standard deviation in all bin ranges except $q^{2} \in[0.1,0.98]$. The error band associated with this LFU parameter $R_{f_{2}^{\prime}}$ is almost zero.

(vi) The $Q$ parameters $\left(\left\langle Q_{F_{L}}\right\rangle,\left\langle Q_{A_{F B}}\right\rangle\right.$, and $\left.\left\langle Q_{5}^{\prime}\right\rangle\right)$. - We provide the SM values and the NP contributions for each $q^{2}$ bin region in the bottom panel in Fig. 2 correspondingly. We observe that, in all $Q_{i}\left(Q_{F_{L}}\right.$, $\left.Q_{A_{F B}}, Q_{5}^{\prime}\right)$ parameters, the predictions in the presence of light $Z^{\prime}$ deviates significantly from the $\mathrm{SM}$ values. For $Q_{F_{L}}$, specifically in the bin region
$[0.1,0.98]$ and $[1.1,6.0]$, we get more than $3 \sigma$ standard deviation, whereas in the rest of the bin rooms it is less than $3 \sigma$ from the SM contribution. Similarly, in the $Q_{A_{F B}}$ observable, we get (3-5) $\sigma$ deviation in all $q^{2}$ bins. From Table III, one can observe clearly for another LFU parameter $Q_{5}^{\prime}$ that it varies (4-9) $\sigma$ deviation from the $\mathrm{SM}$ in all bins enveloped in $q^{2} \in[0.1,6.0]$.

\section{Analysis of $B \rightarrow K_{2}^{*}(1430) \ell^{+} \ell^{-}$in $S M$ and beyond}

Similar to the $B_{s} \rightarrow f_{2}^{\prime} \ell^{+} \ell^{-}$process, we also probe the semileptonic B meson decay to another tensor meson $K_{2}^{*}(1430)$ in the final state which also mediates $b \rightarrow$ sel flavor changing neutral current transition. Here, we also study the variation of the various observables such as BR, $F_{L}, A_{F B}, P_{5}^{\prime}$, and the LFU-sensitive observables $R_{K_{2}^{*}}$, $Q_{F_{L}}, Q_{A_{F B}}$, and $Q_{5}^{\prime}$ both in the SM as well as in the presence of the light $Z^{\prime}$ model in Fig. 3, where $1 \sigma$ error to the SM contribution due to the form factor and CKM element have been considered. In addition to this, we display the corresponding bin plots in Fig. 4. We report the numerical results for all the observables at different $q^{2}$ bin regions in Table IV. We give details of our inspection as below.

Description of the color inputs for the following plots:

Distribution plot.-Black dotted line, SM contribution; green band, $1 \sigma$ error band due to form factors and CKM element; magenta dotted line, light $Z^{\prime}$ contribution.

Binwise plot.-Black bins, SM central values; magenta band, $1 \sigma$ uncertainty due to form factors and CKM element; cyan bin, light $Z^{\prime}$ contribution.

(i) Branching ratio $(B R)$.-We observe the $q^{2}$ behavior in the differential branching ratio of the $B \rightarrow$ $K_{2}^{*} \ell^{+} \ell^{-}$process both in SM as well as in the NP scenario that is displayed in the top-left panel in Fig. 3. Not being significant, the observable in the presence of the NP coupling is reduced in comparison to the SM values. In all bin regions, the observable spans less than $1 \sigma$ deviation from the SM predictions.

(ii) Forward-backward asymmetry $\left(A_{F B}\right)$.-We observe the zero crossing point of the observable $A_{F B}\left(q^{2}\right)$ in the $\mathrm{SM}$ at $\sim 2.8 \mathrm{GeV}^{2}$, whereas it shifted to higher value at $\sim 3.5 \mathrm{GeV}^{2}$ in the presence of NP coupling. The light $Z^{\prime}$ contribution is clearly distinguishable in the range $q^{2} \in[2.5,4]$ and $[1.1,6]$ with $1.15 \sigma$ and $1.09 \sigma$ significance, respectively, whereas less than $1 \sigma$ deviation is observed in the rest of the bin regions.

(iii) Longitudinal polarization fraction $\left(F_{L}\right)$. - In the middle-right panel in Fig. 3, the $q^{2}$ dependency of the longitudinal polarization fraction $F_{L}\left(q^{2}\right)$ suddenly increases up to the peak value at $\sim 1.4 \mathrm{GeV}^{2}$ and then decreases accordingly as the $q^{2}$ value increases. However, it is observed that the peak of 

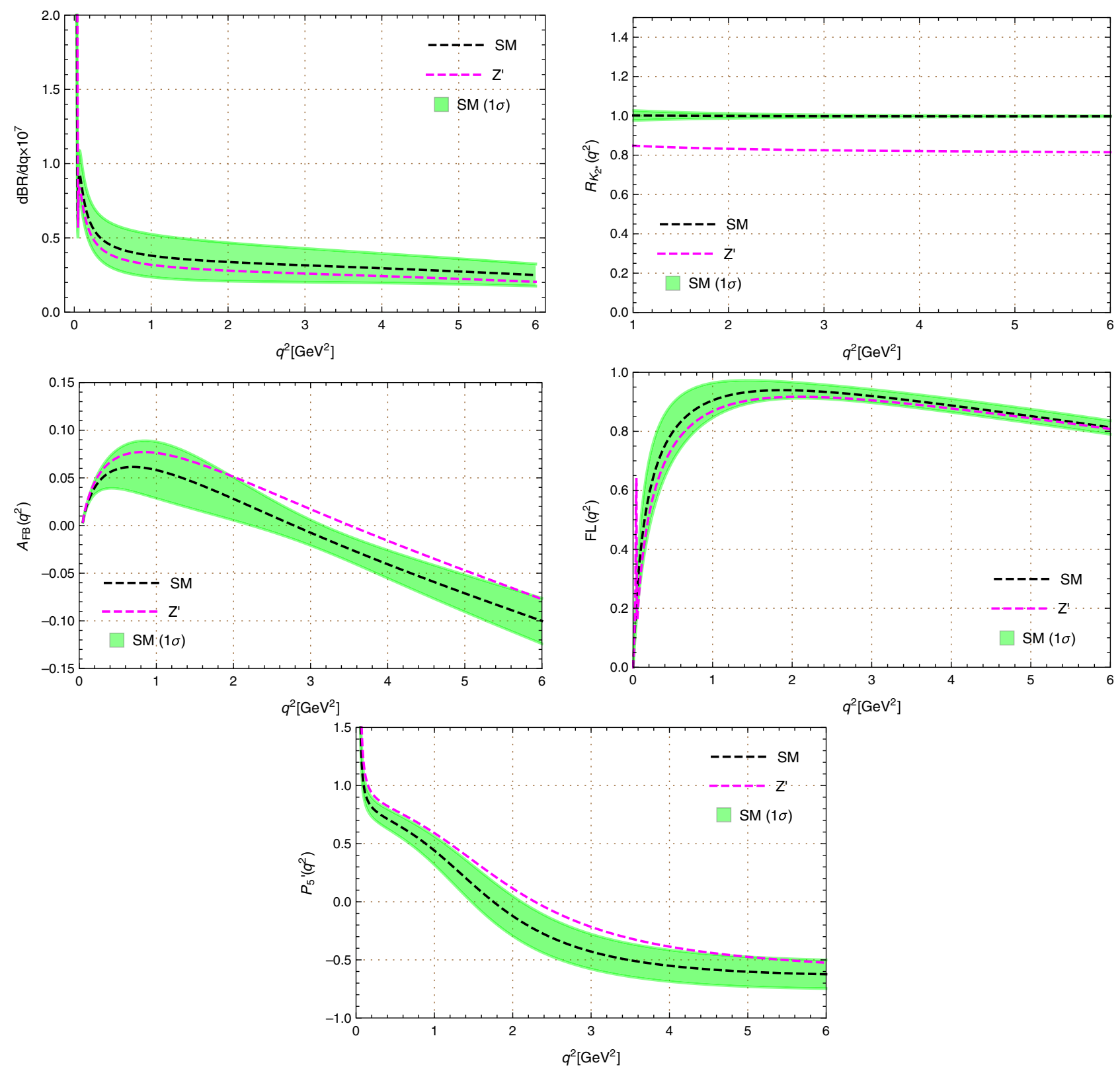

FIG. 3. The $q^{2}$ distribution of various observables such as branching ratio, the polarization fraction, the forward-backward asymmetry, and $P_{5}^{\prime}$ for the $B_{s} \rightarrow K_{2}^{*}(1430) \ell^{+} \ell^{-}$process (black dotted line, SM contribution; green band, $1 \sigma$ uncertainty due to form factors and CKM element; magenta dotted line, $Z^{\prime}$ contribution).

the observable in light $Z^{\prime}$ reduces and is shifted to a lower value than the SM contribution. Here also, no remarkable deviation has been observed in the presence of the NP scenario.

(iv) $P_{5}^{\prime}$. - The angular observable $P_{5}^{\prime}$ is also $q^{2}$ dependent and clearly provides a remarkable contribution in the presence of NP coupling. It is observed that the zero crossing point in the $\mathrm{SM}$ is at $\sim 1.75 \mathrm{GeV}^{2}$, whereas the $Z^{\prime}$ contribution shifts this point to a higher value at $\sim 2.30 \mathrm{GeV}^{2}$.

(v) $L F U$-sensitive parameter $\left(R_{K_{2}^{*}},\left\langle Q_{F_{L}}\right\rangle,\left\langle Q_{A_{F B}}\right\rangle\right.$, and $\left\langle Q_{5}^{\prime}\right\rangle$ ).-In the case of the LFU-sensitive parameter
$R_{K_{2}^{*}}$ shown in the top-right panel in Fig. 3, the observable is quite distinguishable in the presence of the light $Z^{\prime}$ scenario. However, we observe more than $5 \sigma$ deviation than the SM contribution in all $q^{2}$ bin regions starting from 1.1 to $6 \mathrm{GeV}^{2}$, whereas, in the range $q^{2} \in[0.1,0.98], 1.92 \sigma$ significance is observed for this observable. Like $R_{K_{2}^{*}}$, the $Q_{i}$ parameters significantly deviate from the SM. For $Q_{A_{F B}}$, the $Z^{\prime}$ contribution provides (2-4) $\sigma$ deviation in all bin regions as compared to the SM contribution. Similarly, we notice $3.37 \sigma$ standard deviation in the bin range $[0.1,0.98]$ and $<3 \sigma$ in all other $q^{2}$ bin 

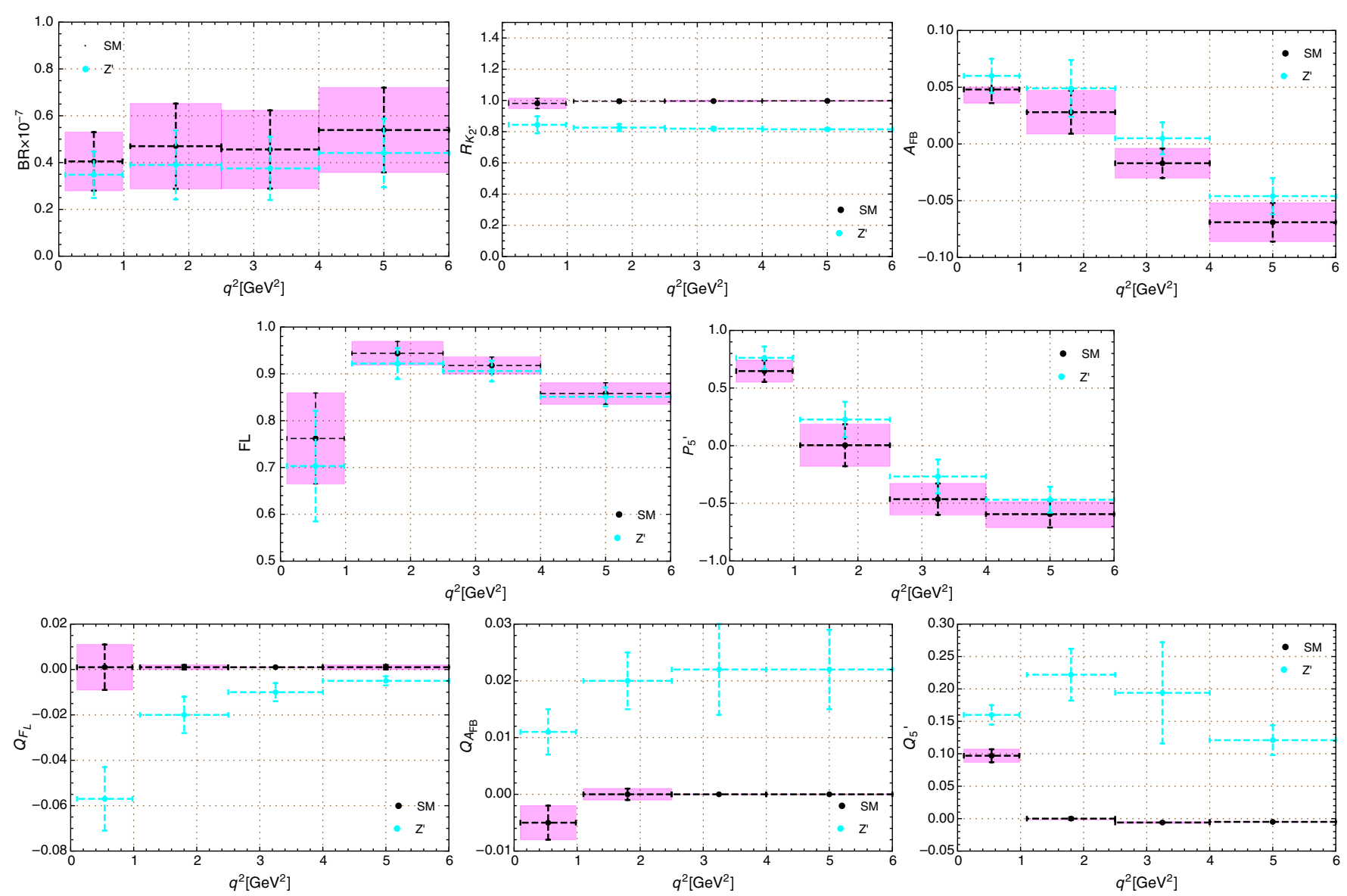

FIG. 4. The binwise distributions of observables such as branching ratio, the polarization fraction, the forward-backward asymmetry, $P_{5}^{\prime}$, and the sensitive LFU parameters $R_{K_{2}^{*}}, Q_{F_{L}}, Q_{A_{F B}}$, and $Q_{5}^{\prime}$ of $B \rightarrow K_{2}^{*} \ell^{+} \ell^{-}$processes (black bins, SM central values; magenta band, $1 \sigma$ uncertainty due to form factors and CKM element; cyan bin, $Z^{\prime}$ contribution).

TABLE IV. Prediction of various observables with $1 \sigma$ standard deviation in SM and $Z^{\prime}$ model for the $B_{s} \rightarrow f_{2}^{\prime} \ell^{+} \ell^{-}$process in different bin rooms.

\begin{tabular}{lcccccc}
\hline \hline Observable & \multicolumn{7}{c}{$[0.10,0.98]$} & {$[1.1,2.5]$} & {$[2.5,4.0]$} & {$[4.0,6.0]$} & {$[1.1,6.0]$} \\
\hline \multicolumn{7}{c}{$B \rightarrow K_{2}^{*} \mu^{+} \mu^{-}$} \\
\hline $\mathcal{B R} \times 10^{-7}$ & $S M$ & $0.405 \pm 0.125$ & $0.470 \pm 0.182$ & $0.456 \pm 0.167$ & $0.539 \pm 0.181$ & $1.467 \pm 0.531$ \\
& $Z^{\prime}$ & $0.348 \pm 0.099$ & $0.390 \pm 0.147$ & $0.375 \pm 0.135$ & $0.441 \pm 0.146$ & $1.208 \pm 0.428$ \\
$\mathcal{A}_{\mathcal{F B}}$ & $S M$ & $0.048 \pm 0.012$ & $0.028 \pm 0.019$ & $-0.017 \pm 0.013$ & $-0.069 \pm 0.017$ & $-0.021 \pm 0.012$ \\
& $Z^{\prime}$ & $0.060 \pm 0.015$ & $0.049 \pm 0.025$ & $0.005 \pm 0.014$ & $-0.046 \pm 0.016$ & $0.000 \pm 0.015$ \\
$\mathcal{F}_{\mathcal{L}}$ & $S M$ & $0.762 \pm 0.097$ & $0.944 \pm 0.025$ & $0.918 \pm 0.018$ & $0.858 \pm 0.023$ & $0.904 \pm 0.026$ \\
& $Z^{\prime}$ & $0.703 \pm 0.118$ & $0.922 \pm 0.033$ & $0.906 \pm 0.022$ & $0.851 \pm 0.020$ & $0.891 \pm 0.030$ \\
$\mathcal{P}_{5}^{\prime}$ & $S M$ & $0.647 \pm 0.094$ & $0.004 \pm 0.182$ & $-0.464 \pm 0.136$ & $-0.594 \pm 0.116$ & $-0.395 \pm 0.128$ \\
& $Z^{\prime}$ & $0.762 \pm 0.098$ & $0.227 \pm 0.153$ & $-0.267 \pm 0.147$ & $-0.469 \pm 0.112$ & $-0.213 \pm 0.145$ \\
$\mathcal{R}_{e}^{\mu}$ & $S M$ & $0.981 \pm 0.033$ & $0.995 \pm 0.011$ & $0.996 \pm 0.004$ & $0.997 \pm 0.002$ & $0.996 \pm 0.006$ \\
& $Z^{\prime}$ & $0.844 \pm 0.054$ & $0.826 \pm 0.023$ & $0.819 \pm 0.011$ & $0.815 \pm 0.007$ & $0.820 \pm 0.008$ \\
$Q_{\mathcal{A}_{\mathcal{B}}}$ & $S M$ & $-0.005 \pm 0.003$ & $-0.000 \pm 0.001$ & $0.000 \pm 0.000$ & $0.000 \pm 0.000$ & $-0.000 \pm 0.000$ \\
$Q_{\mathcal{F}_{\mathcal{L}}}$ & $Z^{\prime}$ & $0.011 \pm 0.004$ & $0.020 \pm 0.005$ & $0.022 \pm 0.008$ & $0.022 \pm 0.007$ & $0.022 \pm 0.008$ \\
$Q_{5}^{\prime}$ & $S M$ & $0.001 \pm 0.010$ & $0.001 \pm 0.001$ & $0.001 \pm 0.000$ & $0.001 \pm 0.001$ & $0.001 \pm 0.001$ \\
& $Z^{\prime}$ & $-0.057 \pm 0.014$ & $-0.020 \pm 0.008$ & $-0.010 \pm 0.004$ & $-0.005 \pm 0.002$ & $-0.011 \pm 0.005$ \\
\hline \hline & $S M$ & $0.097 \pm 0.010$ & $-0.000 \pm 0.002$ & $-0.006 \pm 0.001$ & $-0.005 \pm 0.000$ & $-0.006 \pm 0.001$ \\
& $Z^{\prime}$ & $0.160 \pm 0.015$ & $0.222 \pm 0.040$ & $0.194 \pm 0.078$ & $0.121 \pm 0.023$ & $0.177 \pm 0.052$ \\
\hline
\end{tabular}


ranges for the observable $Q_{F_{L}}$. Last but not the least, the parameter $Q_{5}^{\prime}$ can be observed with more than $5 \sigma$ in the region $q^{2} \in([1.1,2.5],[4,6])$, whereas $3.49 \sigma$ and $3.518 \sigma$ in the regions $[0.1,0.98]$ and $[1.1,6.0]$, respectively. However, in the $q^{2} \in[2.5,4]$ region, we get $2.56 \sigma$ deviation from the SM contribution. The binwise plots for all the above discussed observables are shown in Fig. 4.

\section{CONCLUSION}

Inspired by the anomalies present in $B \rightarrow\left(K, K^{*}\right) \ell^{+} \ell^{-}$ and $B_{s} \rightarrow \phi \mu^{+} \mu^{-}$decays proceeding via $b \rightarrow s \ell^{+} \ell^{-}$flavor changing neutral current quark-level interaction, we scrutinize the semileptonic decays of $B \rightarrow K_{2}^{*}(1430)$ and $B_{s} \rightarrow f_{2}^{\prime}(1525)$ with the charged leptons $(\ell=\mu, e)$ in the presence of the SM and the light $Z^{\prime}$ model. Assuming the NP present in muon mode of a lepton pair in the final state, we constrain the NP coupling by considering the experimental data associated with the clean observable $R_{K}$ in the range $1.1<q^{2}<6.0 \mathrm{GeV}^{2}$ and $R_{K^{*}}$ in the central $q^{2}$ region $[1.1,6.0]$ with the performance of $\chi^{2}$ fit. In the presence of an effective Hamiltonian for $b \rightarrow s \ell \ell$ transition, we provide a detailed study of the behavior of various physical observables such as differential branching ratio, lepton polarization fraction, forward-backward asymmetry, the angular observable $P_{5}^{\prime}$, and LFU-sensitive parameter as the ratio of branching ratios in $B \rightarrow K_{2}^{*}$ and $B_{s} \rightarrow f_{2}^{\prime}$ transition with $\mu$ mode to e mode in the final state in the SM as well as in the presence of light $Z^{\prime}$. The other observables that are very sensitive to lepton flavor universality also draw attention to probe on a few $Q_{i}$ parameters corresponding to the longitudinal polarization fraction $\left(Q_{F_{L}}\right)$, forward-backward asymmetry $\left(Q_{A_{F B}}\right)$, and the angular observable $P_{5}^{\prime}\left(Q_{5}^{\prime}\right)$. With the $q^{2}$-dependent
NP coupling, we give the integrated predictions of all the above discussed prominent observables pertaining to $B \rightarrow K_{2}^{*} \ell^{+} \ell^{-}$and $B_{s} \rightarrow f_{2}^{\prime} \ell^{+} \ell^{-}$decays at different $q^{2}$ bin regions that are compatible with the $\mathrm{LHCb}$ experiment. In this study, all the observables are investigated by considering the form factors obtained from light-cone sum rule approach.

We observed in our analysis that the differential branching ratio is reduced as compared to the SM and notice no significant deviation for this observable in both exclusive $B \rightarrow K_{2}^{*} \ell^{+} \ell^{-}$and $B_{s} \rightarrow f_{2}^{\prime} \ell^{+} \ell^{-}$processes in the presence of a light $Z^{\prime}$ boson. In the observables, the longitudinal fraction and the angular observable $P_{5}^{\prime}$, we get a remarkable contribution in the new physics analysis in both the decay modes. The deviations observed at the LFU parameters such as $R_{f_{2}^{\prime}}$ and $R_{K_{2}^{*}}$ are clearly distinguishable, and, as a complementary decay channel, both can provide an insight into the $R_{f_{2}^{\prime}}$ and $R_{K_{2}^{*}}$ anomalies which could be observed in the LHCb experiment. On the other hand, we also look into the $Q_{i}$ parameters which are very sensitive to LFUV and found that all the observables have profound deviations from the SM contribution. As the $B \rightarrow K_{2}^{*} \ell^{+} \ell^{-}$and $B_{s} \rightarrow f_{2}^{\prime} \ell^{+} \ell^{-}$decay processes have received less attention unlike $B \rightarrow\left(K, K^{*}\right) \ell^{+} \ell^{-}$and $B_{s} \rightarrow \phi \mu^{+} \mu^{-}$decays mediated by $b \rightarrow s \ell \ell$ quark-level transition, it is very important to acquire more data samples from the experiments in order to understand the significance of new physics contributions.

\section{ACKNOWLEDGMENTS}

M. K. M. acknowledges INSPIRE fellowship Division, Department of Science and Technology, Government of India for the nancial support with ID No. 160303.
[1] R. Aaij et al. (LHCb Collaboration), Measurement of $C P$ Averaged Observables in the $B^{0} \rightarrow K^{* 0} \mu^{+} \mu^{-}$Decay, Phys. Rev. Lett. 125, 011802 (2020).

[2] R. Aaij et al. (LHCb Collaboration), Test of lepton universality with $B^{0} \rightarrow K^{* 0} \ell^{+} \ell^{-}$decays, J. High Energy Phys. 08 (2017) 055.

[3] A. Abdesselam et al. (Belle Collaboration), Test of LeptonFlavor Universality in $B \rightarrow K^{*} \ell^{+} \ell^{-}$Decays at Belle, Phys. Rev. Lett. 126, 161801 (2021).

[4] M. Bordone, G. Isidori, and A. Pattori, On the standard model predictions for $R_{K}$ and $R_{K^{*}}$, Eur. Phys. J. C 76, 440 (2016).

[5] G. Hiller and F. Kruger, More model-independent analysis of $b \rightarrow s$ processes, Phys. Rev. D 69, 074020 (2004).
[6] G. Hiller and M. Schmaltz, $R_{K}$ and future $b \rightarrow s \ell \ell$ physics beyond the standard model opportunities, Phys. Rev. D 90, 054014 (2014).

[7] R. Aaij et al. (LHCb Collaboration), Test of lepton universality in beauty-quark decays, arXiv:2103.11769.

[8] R. Aaij et al. (LHCb Collaboration), Measurement of Form-Factor-Independent Observables in the Decay $B^{0} \rightarrow$ $K^{* 0} \mu^{+} \mu^{-}$, Phys. Rev. Lett. 111, 191801 (2013).

[9] R. Aaij et al. (LHCb Collaboration), Angular analysis of the $B^{0} \rightarrow K^{* 0} \mu^{+} \mu^{-}$decay using $3 \mathrm{fb}^{-1}$ of integrated luminosity, J. High Energy Phys. 02 (2016) 104.

[10] M. Aaboud et al. (ATLAS Collaboration), Angular analysis of $B_{d}^{0} \rightarrow K^{*} \mu^{+} \mu^{-}$decays in $p p$ collisions at $\sqrt{s}=8 \mathrm{TeV}$ with the ATLAS detector, J. High Energy Phys. 10 (2018) 047. 
[11] CMS Collaboration, Measurement of the $P_{1}$ and $P_{5}^{\prime}$ angular parameters of the decay $\mathrm{B}^{0} \rightarrow \mathrm{K}^{* 0} \mu^{+} \mu^{-}$in proton-proton collisions at $\sqrt{s}=8 \mathrm{TeV}$, Report No. CMS-PAS-BPH15-008.

[12] A. Abdesselam et al. (Belle Collaboration), Angular analysis of $B^{0} \rightarrow K^{*}(892)^{0} \ell^{+} \ell^{-}$, arXiv:1604.04042.

[13] S. Descotes-Genon, L. Hofer, J. Matias, and J. Virto, On the impact of power corrections in the prediction of $B \rightarrow K^{*} \mu^{+} \mu^{-}$observables, J. High Energy Phys. 12 (2014) 125.

[14] S. Descotes-Genon, T. Hurth, J. Matias, and J. Virto, Optimizing the basis of $B \rightarrow K^{*} l l$ observables in the full kinematic range, J. High Energy Phys. 05 (2013) 137.

[15] A. Bharucha, D. M. Straub, and R. Zwicky, $B \rightarrow V \ell^{+} \ell^{-}$in the standard model from light-cone sum rules, J. High Energy Phys. 08 (2016) 098.

[16] J. Aebischer, J. Kumar, P. Stangl, and D. M. Straub, A global likelihood for precision constraints and flavour anomalies, Eur. Phys. J. C 79, 509 (2019).

[17] M. Tanabashi et al. (Particle Data Group), Review of particle physics, Phys. Rev. D 98, 030001 (2018).

[18] I. Ahmed, M. J. Aslam, M. Junaid, and S. Shafaq, Model independent analysis of B $\rightarrow K_{2}^{*}(1430) \mu^{+} \mu^{-}$decay, J. High Energy Phys. 02 (2012) 045.

[19] S. Rai Choudhury, A. S. Cornell, G. C. Joshi, and B. H. J. McKellar, Analysis of the $B \rightarrow K_{2}^{*}(\rightarrow K \pi) l^{+} l^{-}$decay, Phys. Rev. D 74, 054031 (2006).

[20] H. Hatanaka and K. C. Yang, Radiative and semileptonic B decays involving the tensor meson $\mathrm{K}(2) *(1430)$ in the standard model and beyond, Phys. Rev. D 79, 114008 (2009).

[21] H. Hatanaka and K. C. Yang, Radiative and semileptonic B decays involving higher K-resonances in the final states, Eur. Phys. J. C 67, 149 (2010).

[22] M. Junaid, M. J. Aslam, and I. Ahmed, Complementarity of semileptonic $B$ to $K_{2}^{*}(1430)$ and $K^{*}(892)$ decays in the standard model with fourth generation, Int. J. Mod. Phys. A 27, 1250149 (2012).

[23] C. D. Lu and W. Wang, Analysis of $B \rightarrow K_{J}^{*}(\rightarrow K \pi) \mu^{+} \mu^{-}$in the higher kaon resonance region, Phys. Rev. D 85, 034014 (2012).

[24] T. M. Aliev and M. Savci, $B \rightarrow K_{2} \ell^{+} \ell^{-}$decay beyond the standard model, Phys. Rev. D 85, 015007 (2012).

[25] D. Das, B. Kindra, G. Kumar, and N. Mahajan, $B \rightarrow$ $K_{2}^{*}(1430) \ell^{+} \ell^{-}$distributions at large recoil in the standard model and beyond, Phys. Rev. D 99, 093012 (2019).

[26] R. H. Li, C. D. Lu, and W. Wang, Branching ratios, forwardbackward asymmetries and angular distributions of $B \rightarrow$ $K_{2}^{*} l^{+} l^{-}$in the standard model and new physics scenarios, Phys. Rev. D 83, 034034 (2011).

[27] N. Rajeev, N. Sahoo, and R. Dutta, Angular analysis of $B_{s} \rightarrow f_{2}^{\prime}(1525)\left(\rightarrow K^{+} K^{-}\right) \mu^{+} \mu^{-}$decays as a probe to lepton flavor universality violation, Phys. Rev. D 103, 095007 (2021).

[28] N. Isgur, D. Scora, B. Grinstein, and M. B. Wise, Semileptonic B and D decays in the quark model, Phys. Rev. D 39, 799 (1989).

[29] D. Scora and N. Isgur, Semileptonic meson decays in the quark model: An update, Phys. Rev. D 52, 2783 (1995).
[30] N. Sharma and R. C. Verma, Predictions of Bc meson decay emitting pseudoscalar and heavy scalar mesons using ISGW II model, Phys. Rev. D 82, 094014 (2010).

[31] W. Wang, B to tensor meson form factors in the perturbative QCD approach, Phys. Rev. D 83, 014008 (2011).

[32] K. C. Yang, B to light tensor meson form factors derived from light-cone sum rules, Phys. Lett. B 695, 444 (2011).

[33] A. K. Alok, B. Bhattacharya, A. Datta, D. Kumar, J. Kumar, and D. London, New physics in $b \rightarrow s \mu^{+} \mu^{-}$after the measurement of $R_{K^{*}}$, Phys. Rev. D 96, 095009 (2017).

[34] A. K. Alok, B. Bhattacharya, D. Kumar, J. Kumar, D. London, and S. U. Sankar, New physics in $b \rightarrow s \mu^{+} \mu^{-}$: Distinguishing models through $C P$-violating effects, Phys. Rev. D 96, 015034 (2017).

[35] W. Altmannshofer, P. Stangl, and D. M. Straub, Interpreting hints for lepton flavor universality violation, Phys. Rev. D 96, 055008 (2017).

[36] G. Hiller and I. Nisandzic, $R_{K}$ and $R_{K^{*}}$ beyond the standard model, Phys. Rev. D 96, 035003 (2017).

[37] B. Capdevila, A. Crivellin, S. Descotes-Genon, J. Matias, and J. Virto, Patterns of new physics in $b \rightarrow s \ell^{+} \ell^{-}$ transitions in the light of recent data, J. High Energy Phys. 01 (2018) 093.

[38] F. Sala and D. M. Straub, A new light particle in B decays?, Phys. Lett. B 774, 205 (2017).

[39] M. Ciuchini, A. M. Coutinho, M. Fedele, E. Franco, A. Paul, L. Silvestrini, and M. Valli, On flavourful easter eggs for new physics hunger and lepton flavour universality violation, Eur. Phys. J. C 77, 688 (2017).

[40] G. D’Amico, M. Nardecchia, P. Panci, F. Sannino, A. Strumia, R. Torre, and A. Urbano, Flavour anomalies after the $R_{K^{*}}$ measurement, J. High Energy Phys. 09 (2017) 010.

[41] L. S. Geng, B. Grinstein, S. Jäger, J. Martin Camalich, X. L. Ren, and R.X. Shi, Towards the discovery of new physics with lepton-universality ratios of $b \rightarrow s \ell \ell$ decays, Phys. Rev. D 96, 093006 (2017).

[42] S. Di Chiara, A. Fowlie, S. Fraser, C. Marzo, L. Marzola, M. Raidal, and C. Spethmann, Minimal flavor-changing $Z^{\prime}$ models and muon $g-2$ after the $R_{K^{*}}$ measurement, Nucl. Phys. B923, 245 (2017).

[43] A. Datta, J. Kumar, J. Liao, and D. Marfatia, New light mediators for the $R_{K}$ and $R_{K^{*}}$ puzzles, Phys. Rev. D 97 , 115038 (2018).

[44] A. Datta, J. Liao, and D. Marfatia, A light $Z^{\prime}$ for the $R_{K}$ puzzle and nonstandard neutrino interactions, Phys. Lett. B 768, 265 (2017).

[45] D. Ghosh, Explaining the $R_{K}$ and $R_{K^{*}}$ anomalies, Eur. Phys. J. C 77, 694 (2017).

[46] F. Bishara, U. Haisch, and P. F. Monni, Regarding light resonance interpretations of the B decay anomalies, Phys. Rev. D 96, 055002 (2017).

[47] A. J. Buras and M. Munz, Effective Hamiltonian for $B \rightarrow$ $X_{s} e^{+} e^{-}$beyond leading logarithms in the NDR and HV schemes, Phys. Rev. D 52, 186 (1995).

[48] M. Misiak, The $b \rightarrow s e^{+} e^{-}$and $b \rightarrow s \gamma$ decays with nextto-leading logarithmic QCD corrections, Nucl. Phys. B393, 23 (1993); B439, 461(E) (1995).

[49] A. Khodjamirian, T. Mannel, A. A. Pivovarov, and Y. M. Wang, Charm-loop effect in $B \rightarrow K^{(*)} \ell^{+} \ell^{-}$and $B \rightarrow K^{*} \gamma$, J. High Energy Phys. 09 (2010) 089. 
[50] N. Gubernari, D. van Dyk, and J. Virto, Non-local matrix elements in $B_{(s)} \rightarrow\left\{K^{(*)}, \phi\right\} \ell^{+} \ell^{-}, \mathrm{J}$. High Energy Phys. 02 (2021) 088.

[51] E. R. Berger, A. Donnachie, H. G. Dosch, and O. Nachtmann, Observing the odderon: Tensor meson photoproduction, Eur. Phys. J. C 14, 673 (2000).

[52] Y. B. Zuo, C. X. Yue, B. Yu, Y. H. Kou, Y. Chen, and W. Ling, $B_{(s)}$ to light tensor meson form factors via LCSR in HQEFT with applications to semileptonic decays, Eur. Phys. J. C 81, 30 (2021).

[53] L. Calibbi, A. Crivellin, and T. Ota, Effective Field Theory Approach to $b \rightarrow s \ell \ell^{(')}, B \rightarrow K^{(*)} \nu \bar{\nu}$ and $B \rightarrow D^{(*)} \tau \nu$ with Third Generation Couplings, Phys. Rev. Lett. 115, 181801 (2015).

[54] A. Crivellin, G. D'Ambrosio, and J. Heeck, Addressing the LHC flavor anomalies with horizontal gauge symmetries, Phys. Rev. D 91, 075006 (2015).

[55] S. M. Boucenna, A. Celis, J. Fuentes-Martin, A. Vicente, and J. Virto, Non-Abelian gauge extensions for B-decay anomalies, Phys. Lett. B 760, 214 (2016).

[56] M. Huschle et al. (Belle Collaboration), Measurement of the branching ratio of $\bar{B} \rightarrow D^{(*)} \tau^{-} \bar{\nu}_{\tau}$ relative to $\bar{B} \rightarrow D^{(*)} \ell^{-} \bar{\nu}_{\ell}$ decays with hadronic tagging at Belle, Phys. Rev. D 92, 072014 (2015).

[57] R. Aaij et al. (LHCb Collaboration), Measurement of the Ratio of Branching Fractions $\mathcal{B}\left(\bar{B}^{0} \rightarrow D^{*+} \tau^{-} \bar{\nu}_{\tau}\right) /$ $\mathcal{B}\left(\bar{B}^{0} \rightarrow D^{*+} \mu^{-} \bar{\nu}_{\mu}\right)$, Phys. Rev. Lett. 115, 111803 (2015); 115, 159901(E) (2015).

[58] M. K. Mohapatra, N. Rajeev, and R. Dutta, Combined analysis of $B_{c} \rightarrow D_{s}^{(*)} \mu^{+} \mu^{-}$and $B_{c} \rightarrow D_{s}^{(*)} \nu \bar{\nu}$ decays within $Z^{\prime}$ and leptoquark new physics models, arXiv:2108.10106.

[59] A. J. Buras and J. Girrbach, Left-handed Z' and Z FCNC quark couplings facing new $b \rightarrow s \mu^{+} \mu^{-}$data, J. High Energy Phys. 12 (2013) 009.
[60] R. Gauld, F. Goertz, and U. Haisch, Minimal Z' explanations of the $B \rightarrow K^{*} \mu^{+} \mu^{-}$anomaly, Phys. Rev. D 89, 015005 (2014).

[61] R. Gauld, F. Goertz, and U. Haisch, An explicit Z'-boson explanation of the $B \rightarrow K^{*} \mu^{+} \mu^{-}$anomaly, J. High Energy Phys. 01 (2014) 069.

[62] C. W. Chiang, X. G. He, and G. Valencia, Z' model for $b \rightarrow$ $s \ell \bar{\ell}$ flavor anomalies, Phys. Rev. D 93, 074003 (2016).

[63] A. Crivellin, L. Hofer, J. Matias, U. Nierste, S. Pokorski, and J. Rosiek, Lepton-flavor violating B decays in generic $\mathrm{Z}^{\prime}$ modelsPhys. Rev. D 92, 054013 (2015).

[64] B. Abi et al. (Muon g-2 Collaboration), Measurement of the Positive Muon Anomalous Magnetic Moment to 0.46 ppm, Phys. Rev. Lett. 126, 141801 (2021).

[65] G. W. Bennett et al. (Muon g-2 Collaboration), Final report of the muon E821 anomalous magnetic moment measurement at BNL, Phys. Rev. D 73, 072003 (2006).

[66] T. Aoyama, N. Asmussen, M. Benayoun, J. Bijnens, T. Blum, M. Bruno, I. Caprini, C. M. Carloni Calame, M. Cè, and G. Colangelo et al., The anomalous magnetic moment of the muon in the standard model, Phys. Rep. 887, 1 (2020).

[67] J. P. Leveille, The second order weak correction to (G-2) of the muon in arbitrary gauge models, Nucl. Phys. B137, 63 (1978).

[68] P. A. Zyla et al. (Particle Data Group), Review of particle physics, Prog. Theor. Exp. Phys. 2020, 083 C01 (2020).

[69] W. Altmannshofer, P. Ball, A. Bharucha, A. J. Buras, D. M. Straub, and M. Wick, Symmetries and asymmetries of $B \rightarrow$ $K^{*} \mu^{+} \mu^{-}$Decays in the standard model and beyond, J. High Energy Phys. 01 (2009) 019.

[70] A. Ali, P. Ball, L. T. Handoko, and G. Hiller, A Comparative study of the decays $B \rightarrow\left(K, K^{*}\right) \ell^{+} \ell^{-}$in standard model and supersymmetric theories, Phys. Rev. D 61, 074024 (2000). 